\title{
Compare the efficacy and safety of programmed cell death-1 (PD-1) and programmed cell death ligand-1 (PD-L1) inhibitors for advanced non-small cell lung cancer: a Bayesian analysis
}

\author{
Jiaqi Liang ${ }^{1 \#}$, Ming Li ${ }^{1 \#}$, Qihai Sui ${ }^{1,2 \#}$, Zhengyang Hu${ }^{1}$, Yunyi Bian ${ }^{1}$, Yiwei Huang ${ }^{1}$, Cheng Zhan ${ }^{1}$, Wei Jiang ${ }^{1}$, \\ Qun Wang ${ }^{1}$, Lijie Tan ${ }^{1}$ \\ ${ }^{1}$ Department of Thoracic Surgery, Zhongshan Hospital, Fudan University, Shanghai, China; ${ }^{2}$ Eight-year Program Clinical Medicine, Shanghai \\ Medical College, Fudan University, Shanghai, China \\ Contributions: (I) Conception and design: All authors; (II) Administrative support: All authors; (III) Provision of study materials or patients: All \\ authors; (IV) Collection and assembly of data: All authors; (V) Data analysis and interpretation: All authors; (VI) Manuscript writing: All authors; (VII) \\ Final approval of manuscript: All authors. \\ "These authors contributed equally to this work. \\ Correspondence to: Cheng Zhan; Wei Jiang. Department of Thoracic Surgery, Zhongshan Hospital, Fudan University, 180 Fenglin Road, Shanghai, \\ China. Email: czhan10@fudan.edu.cn; jiang.wei1@zs-hospital.sh.cn.
}

Background: Inhibitors of programmed cell death-1 (PD-1) and its ligand (PD-L1) have represented a novel approach for the management of advanced non-small cell lung cancer (NSCLC). In this study, we aimed to estimate five anti-PD-1/L1 agents (nivolumab, pembrolizumab, atezolizumab, durvalumab, and avelumab) using network meta-analyses (NMAs) and the Bayesian method to provide suggestions for advanced NSCLC treatments.

Methods: We searched PubMed, Web of Science, Embase, and the Wiley Online Library for eligible studies published up to March 2020. Both pairwise analyses and NMAs were conducted with clinical outcomes, including overall survival (OS), progression-free survival (PFS), objective response rate, and the incidences of adverse events. Results were presented in several patient populations according to treatment lines and PD-L1 status.

Results: Nineteen randomized clinical trials (RCTs) involving 11,456 patients were included in our study. PD-1/L1 inhibitors showed significant benefits over chemotherapies in OS regardless of tumor PD-L1 status [first-line settings: $\mathrm{OS}=0.85,95 \% \mathrm{CI}(0.77,0.94), \mathrm{I}^{2}=37 \%$; second- or further-line settings: OS $=0.77$, $95 \%$ CI $\left.(0.71,0.84), I^{2}=37 \%\right]$. The combined regimen of pembrolizumab and chemotherapy stood out to be the most effective and safest for patients in the first-line settings. Pembrolizumab monotherapy was showed to be the best especially for patients with PD-L1 $\geq 50 \%$. In the subsequent-line settings, nivolumab ranked the best in improving the survival of patients, and durvalumab had the greatest effect in tumor shrinkage. Atezolizumab, followed by nivolumab, ranked the safest in reducing adverse events, whereas durvalumab was showed with the largest side effects among the five inhibitors.

Conclusions: The combination of pembrolizumab with chemotherapy is suitable for advanced NSCLC patients who have not received any systematic treatments before, and pembrolizumab monotherapy should also be considered, especially for patients with highly-expressed PD-L1 ( $\geq 50 \%)$. Nivolumab is the best option for patients with advanced NSCLC whose tumors have progressed following chemotherapies or combined modalities of treatments including chemotherapy. However, our results need to be further validated in future head-to-head clinical trials. 
Keywords: Non-small cell lung cancer (NSCLC); checkpoint inhibitors (ICIs); programmed cell death-1 (PD-1); programmed cell death ligand-1 (PD-L1); network meta-analysis (NMA)

Submitted Feb 01, 2020. Accepted for publication Jun 23, 2020.

doi: $10.21037 /$ tlcr-20-192

View this article at: http://dx.doi.org/10.21037/tlcr-20-192

\section{Introduction}

Lung cancer has remained the most common cause of cancer death in recent years, and non-small cell lung cancer (NSCLC) represents $87 \%$ of all cases, including adenocarcinomas and squamous cell carcinomas as its main subtypes (1-4). For advanced NSCLC patients, molecular targeted therapy is considered to be a standard first-line regimen for those with genomic mutations, while platinumbased doublet chemotherapy optimizes treating efficacy for those with unknown mutational status $(5,6)$. Options such as pemetrexed and docetaxel serve as second-line and beyond or maintenance therapy for advanced NSCLC with poor tumor response or disease progression $(7,8)$. Apart from the treatments above, immunotherapy has been a novel approach for advanced NSCLC over the last decade, where the inhibition of immune checkpoints plays a pivotal role, including programmed cell death-1 (PD-1) and its ligand programmed death ligand-1 (PD-L1) $(9,10)$. Nivolumab and pembrolizumab are PD-1-targeted inhibitors, whereas atezolizumab, durvalumab, as well as avelumab exert their inhibitory potency on PD-L1 (11).

In advanced NSCLC, all of the five above immune checkpoint inhibitors (ICIs) have shown anticancer activity in earlier phase I or II trials, with objective response rates (ORR) varying between $20-40 \%$ (12-16). Phase III randomized clinical trials (RCTs) were subsequently conducted to validate the clinical efficacy of these ICIs, given the promising efficacy obtained in the preceding studies. Patients were reported to benefit from PD-1/L1 inhibitors in almost all late-phase trials, regardless of the treatment line (17-21). When compared to chemotherapy, previous meta-analyses combining these RCTs above have also shown promising survival benefits as well as safety profiles for all three approved inhibitors, including nivolumab, pembrolizumab, and atezolizumab (22-25). An indirect analysis has shown therapeutic advantages of PD-1 inhibitors, nivolumab and pembrolizumab, over PD-L1 inhibitor, atezolizumab, in pretreated patients with NSCLC (26), but the evidence is still lacking and there is still a lack of head-to-head comparisons to directly determine the best agent. In the present study, we performed a systematic review and meta-analysis of several phase II/III RCTs associated with PD-1/L1 inhibitors in advanced NSCLC. The ultimate purpose of this study was to determine the relative efficacy and safety of the existing PD-1/L1 inhibitors in both the first and subsequent settings. The results provided suggestions for clinicians to develop individualized treatments with PD-1/L1 inhibitors for diverse patients with advanced NSCLC. We present the following article in accordance with the PRISMA reporting checklist (available at http://dx.doi.org/10.21037/tlcr-20-192).

\section{Methods}

\section{Protocol and registration}

The present report has been prepared according to the recommendations of the Cochrane Comparing Multiple Interventions Methods Group and the PRISMA extension statement for systematic reviews incorporating network meta-analyses (NMAs). The protocol of this study was also registered with PROSPERO (ID: CRD 42020163743) (https://www.crd.york.ac.uk/prospero/).

\section{Selection criteria}

Studies were considered if they met the inclusion criteria as follows: (I) prospective RCTs; (II) patients with histologically proven diagnosis of advanced NSCLC; (III) studies comparing the addition or single use of PD-1/ L1 inhibitors with platinum-based chemotherapy among patients with no prior systemic anticancer therapies in the first-line settings; and studies comparing PD-1/L1 inhibitor monotherapy with chemotherapy among patients whose tumors have progressed or recurred following (adjuvant or neo-adjuvant) combined modalities including chemotherapies in the second- or further-line settings; (IV) studies reporting efficacy outcomes, including overall survival (OS), progression-free survival (PFS), and ORR; and $(\mathrm{V})$ studies displayed with safety profiles, including 
the incidence of severe adverse events (SAEs) as well as respiratory and thoracic disorders. Furthermore, the following studies were excluded to minimize the risk of bias: (I) unrandomized studies, retrospective studies, reviews, meta-analysis, case reports, conference reports, letters, or comments; (II) studies in which the comparison drugs were in combination with radiotherapy, other targeted drugs (other than bevacizumab) or other immunotherapy drugs; and (III) studies lacking necessary data or overlapped studies.

\section{Search strategy}

We started to search on the PubMed, Web of Science, Embase, and the Wiley Online Library from August 2019 for eligible studies published up to March 2020, with a language restriction of English. The following groups of keywords and medical terms were used: ("non-small cell lung cancer" or "non-small cell lung carcinoma" or "nonsmall cell lung neoplasms" or "lung adenocarcinoma" or "lung squamous cell carcinoma" or "large cell lung cancer") and ("programmed death 1" or "PD-1" or "programmed death-ligand 1" or "PD-L1" or "immunotherapy" or "immune checkpoint inhibitors" or "PD-1/PD-L1 inhibitors" or "PD-1/PD-L1 blockades" or "anti-PD-1/PDL1" or "nivolumab" or "pembrolizumab" or "atezolizumab" or "durvalumab" or "avelumab"). We manually reviewed the reference lists of relevant studies and tracked the "clinicaltrials.gov" site (https://clinicaltrials.gov/) for the latest updated data on the eligible studies.

\section{Selection process}

A four-phase process was conducted for the selection of studies according to the PRISMA Statement. After searching for records on the websites, studies were screened based on the publication categories specified in the above criteria. Studies were further assessed with full-texts according to their study design and reported outcomes. Eligible ones were finally included if the characteristics of participants, interventions, and outcomes were similar across each study. Two reviewers (Liang and Sui) independently examined publications that met the inclusion criteria. Discrepancies were solved by discussion or adjudication with a third reviewer $(\mathrm{Li})$. Studies were selected with the longest time of follow-up if they had experienced multiple intervals of analysis.

\section{Data extraction}

The parameters for efficacy in this study were OS, PFS as well as ORR, and the incidence of SAEs, as well as the respiratory and thoracic disorders, which were used to analyze the safety profiles. Survival outcomes, OS and PFS, were reported with hazard ratios (HRs) together with 95\% confidence intervals (CIs). Of note, when HRs could not be extracted from the results in the literature, they would be computed by extractions of survival curves using Engauge Digitizer software with a high degree of accuracy according to established methods (27). Other outcomes, ORRs and the incidence of adverse events, were extracted or calculated using the original number of reporting events following established methods (28). General characteristics of outcomes and enrolled patients in the included studies were obtained.

\section{Assessment of risk of bias}

The qualities of included trials were assessed following the instructions of the Cochrane Handbook for Systemic Reviews of Interventions, in which six aspects were evaluated: random sequence generation, allocation concealment, blinding of participants and personnel, blinding of outcome assessment, incomplete outcome data, selective reporting, and other sources of bias (29). Effect account was estimated through sensitive analyses for the impact of included studies with high or unclear risk of bias.

\section{Statistical analysis}

Data were first analyzed through direct pairwise comparisons in all intention-treated patient population, and the results presented with pooled-estimated HRs $\left(\mathrm{HR}_{\text {pooled }}\right)$ together with $95 \%$ CIs. Mixed treatment comparisons incorporating direct and indirect results were subsequently generated within Bayesian frameworks using Markov chain Monte Carlo methods. Analyses above were performed by Review Manager (version 5.3) (https://community.cochrane. org/help/tools-and-software/revman-5) and R (version 3.6.1) as well as R packages (https://www.r-project.org/). In the R package 'gemtc', each chain was fitted with 20,000 iterations. Afterward, different regimens were ranked by efficacy and safety outcomes by calculating the hazard ratios (HRs) or relative risks (RRs) as well as the proportion of iterations of the Markov chain. Ranking probabilities of 
treatments were estimated to determine the likelihood of therapies in a best-to-worst order.

Heterogeneity between studies was measured by the I-square test and $\mathrm{P}$ value. If the I-square value was $\geq 75 \%$, we would regard it as high heterogeneity, and $<25 \%$, as low heterogeneity, while others would remain as medium heterogeneity. The random effect-based model by Der Simonian and Laird was used to perform meta-analyses for studies with high heterogeneity, while the fixed effectbased Mantel-Haenszel model was used for the low and medium heterogeneous models (30,31). All the results were considered to be of significance with a value of $\mathrm{P}<0.05$, and no significance was attributed otherwise. Sensitivity analyses were conducted in case of high heterogeneity ( $\geq 75 \%)$, and studies which would largely affected the heterogeneity were omitted from the model one after another based on the sensitivity results. Before and after the omissions, pooled efficacy and safety together with the statistical significances were compared.

\section{Ethical statements}

Ethical approval was exempted by the Institutional Review Committee of Zhongshan Hospital, Fudan University, Shanghai, China.

\section{Results}

\section{Literature search results}

The literature search identified a total of 249 records, of which 34 were excluded because of duplicates; 127 records were excluded because of reviews, case reports, conference reports, letters, comments or summaries; three records were excluded because of meta-analyses; 28 records were excluded because of non-perspective RCTs; seven records were excluded because of early phases; 10 records were excluded because of the same trial with multiple follow-ups; three records were excluded because the combination of PD-1/L1 inhibitors or chemotherapy was combined with other drugs or therapies (other than bevacizumab); four records were excluded because of mixed diagnosis of patients enrolled; and three records were excluded because of insufficient result posted. A total of 28 trials were assessed as eligible ones and nine studies were excluded because of their heterogeneity and incompatibility for the quantitative analysis.

Finally, 19 studies involving 11,456 participants with advanced NSCLC (eleven studies with PD-1/L1 inhibitors in the first-line settings involving 6,635 patients and eight studies with PD-1/L1 inhibitors in the subsequent settings involving 4,821 patients) met all the inclusion or exclusion criteria and were included in the meta-analysis $(17,19,20,32-44)$. All of the five anti-PD-1/L1 agents were covered in our study. In the included studies, patients were originally stratified into subgroups by the expressing PDL1 status of tumor cells, which was presented by the tumor proportion score (TPS). The cutting-off points of PD-L1 TPS included $1 \%, 5 \%, 10 \%, 25 \%$, and $50 \%$. $1 \%$ and $50 \%$ were finally selected as cut-offs for our subgroup metaanalyses because they were the most intersections for the clinical results reported by different studies that we had included. A PRISMA diagram summarizing our literature search results and selection is shown in Figure 1. The general characteristics of enrolled patients in the included trials, as well as clinical outcomes, are outlined in Tables 1,2. The whole network is illustrated in Figure 2, in which solid lines between the different drugs represent the number of studies, and dashed lines represent indirect comparisons that we can obtain through the network.

\section{Assessment of risk of bias}

Seventeen of all were considered at high risk of bias in performance and detection because they were openlabel. Although only two of these studies (Keynote 189 \& Keynote 407) were double-blind, outcomes on whether patients could benefit from PD-1/L1 inhibitors or not were reported to be similar to those in open-label trials. Nine undergoing studies were at a high risk of attrition bias resulting from the incomplete outcomes. Data on risk of bias of each included study are presented in Figure S1A. Results of any assessment of risk of bias across studies are illustrated in Figure S1B.

\section{Comparisons of regimens}

\section{Drug efficacy \\ First-line settings}

We analyzed three populations of patients in this setting: intention-to-treat (ITT) population as well as patients with PD-L1 TPS $\geq 1 \%$ and TPS $\geq 50 \%$. Sensitivity analyses were performed in the following models with high heterogeneity, and none of the pooled results showed any alteration in the statistical significance after the heterogeneity reduction. According to results of pair-wise comparisons, additions of PD-1/L1 inhibitors to chemotherapy were shown to be 


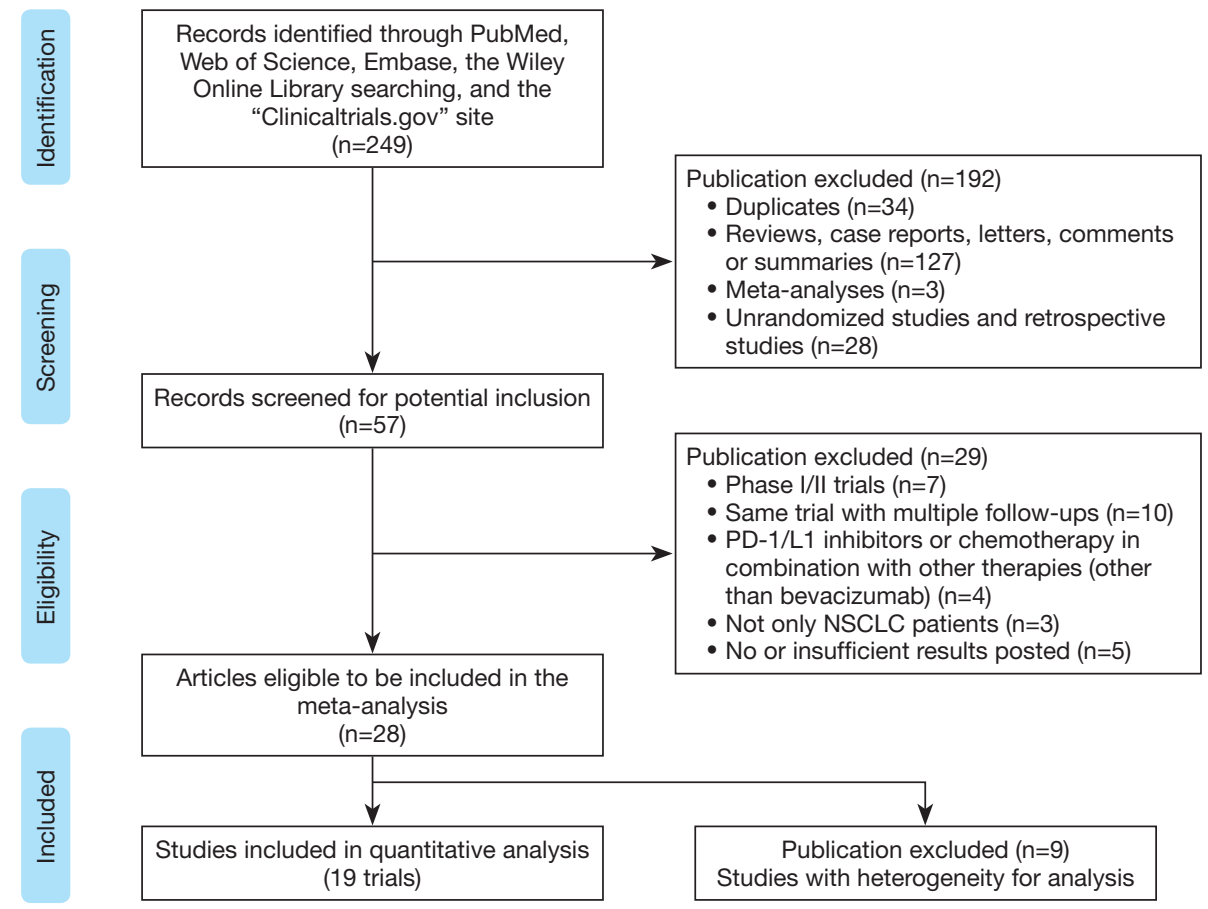

Figure 1 PRISMA diagram of search results and selections.

superior to chemotherapeutic drugs alone in terms of OS [OS HR $\left.\mathrm{Hooled}=0.85 ; 95 \% \mathrm{CI}(0.77,0.94) ; \mathrm{I}^{2}=37 \%\right]$ and $\mathrm{PFS}$ $\left[\mathrm{PFS} \mathrm{HR}_{\text {pooled }}=0.63 ; 95 \% \mathrm{CI}(0.58,0.69) ; \mathrm{I}^{2}=58 \%\right.$ ] (Figure $S 2 A, B)$. Benefits were also significant in terms of ORR [ORR $\left.\mathrm{RR}_{\text {pooled }}=1.30 ; 95 \% \mathrm{CI}(1.17,1.46) ; \mathrm{I}^{2}=71 \%\right]$ (Figure S2C). In the population of patients with PD-L1 TPS $\geq 1 \%$, both the single use of the PD-1/L1 inhibitors and their combination with chemotherapy showed a significantly lower risk of death for patients, compared with chemotherapeutic drugs [OS $\mathrm{HR}_{\text {pooled }}=0.84 ; 95 \%$ CI $(0.76,0.92) ; \mathrm{I}^{2}=49 \%$ ] (Figure $S 2 D)$. However, no benefit was shown in terms of PFS in this subgroup of patients [PFS $\mathrm{HR}_{\text {pooled }}=1.08 ; 95 \%$ CI $\left.(0.98,1.18) ; I^{2}=0 \%\right]$ (Figure $S 2 E$ ). Similar results were also reported among patients treated with nivolumab or pembrolizumab monotherapy with PD-L1 TPS $\geq 50 \%$ [OS $\mathrm{HR}_{\text {pooled }}=0.70 ; 95 \%$ CI $(0.60,0.81) ; \mathrm{I}^{2}=0 \% ; \mathrm{PFS} \mathrm{HR}_{\text {pooled }}$ $=0.87 ; 95 \% \mathrm{CI}(0.75,1.02) ; \mathrm{I}^{2}=0 \%$ ] (Figure $S 2 F, G$ ).

According to the ranking results from NMA, the top three of all regimens in terms of efficacy were pembrolizumab combined with chemotherapy, atezolizumab combined with chemotherapy, and pembrolizumab monotherapy. Pembrolizumab combined therapy seemed to be the most effective with a probability of $84 \%$ ranking first in OS and $90 \%$ in PFS or ORR (Figures $3 A, B, S 3 A$ ) in the ITT patient population. Its superiority to atezolizumab combined therapy was significant both in OS and PFS [OS $\mathrm{HR}_{\text {indirect }}=0.69 ; 95 \% \mathrm{CI}(0.54,0.86) ; \mathrm{I}^{2}=14 \% ; \mathrm{PFS} \mathrm{HR}_{\text {indirect }}$ $=0.77 ; 95 \%$ CI $\left.(0.64,0.93) ; I^{2}=0 \%\right]$ (Table 3). It also ranked as the best among the patient with PD-L1 TPS $\geq 1 \%$, with a probability of $58 \%$ in OS and a probability of $78 \%$ in PFS (Figure 3C,D). The superiority of pembrolizumab combined therapy to pembrolizumab was also both significant in OS and PFS [OS HR indirect $=0.71 ; 95 \%$ CI $(0.56,0.90) ; \mathrm{I}^{2}=11 \%$; PFS HR indirect $\left._{\text {en }}=0.45 ; 95 \% \mathrm{CI}(0.36,0.56) ; \mathrm{I}^{2}=9 \%\right]$ (Table 3).

The combination of atezolizumab and chemotherapy ranked in the second place in both of the ITT population and among patients with PD-L1 TPS $\geq 1 \%$, but there was no significant difference between it and the third-rank pembrolizumab monotherapy, either in terms of PFS or OS. Also, the advantage of pembrolizumab monotherapy was of no significance when compared to the drugs that ranked behind (nivolumab and durvalumab) (Table 3). However, pembrolizumab monotherapy appeared to be the most effective agent with a high probability of $87 \%$ in terms of OS and $81 \%$ of PFS (Figure $S 3 B, C$ ) for patients with PD-L1 TPS $\geq 50 \%$ temporarily, because the lack of efficacy outcomes related to pembrolizumab or atezolizumab combined therapies. The superiority of pembrolizumab to 


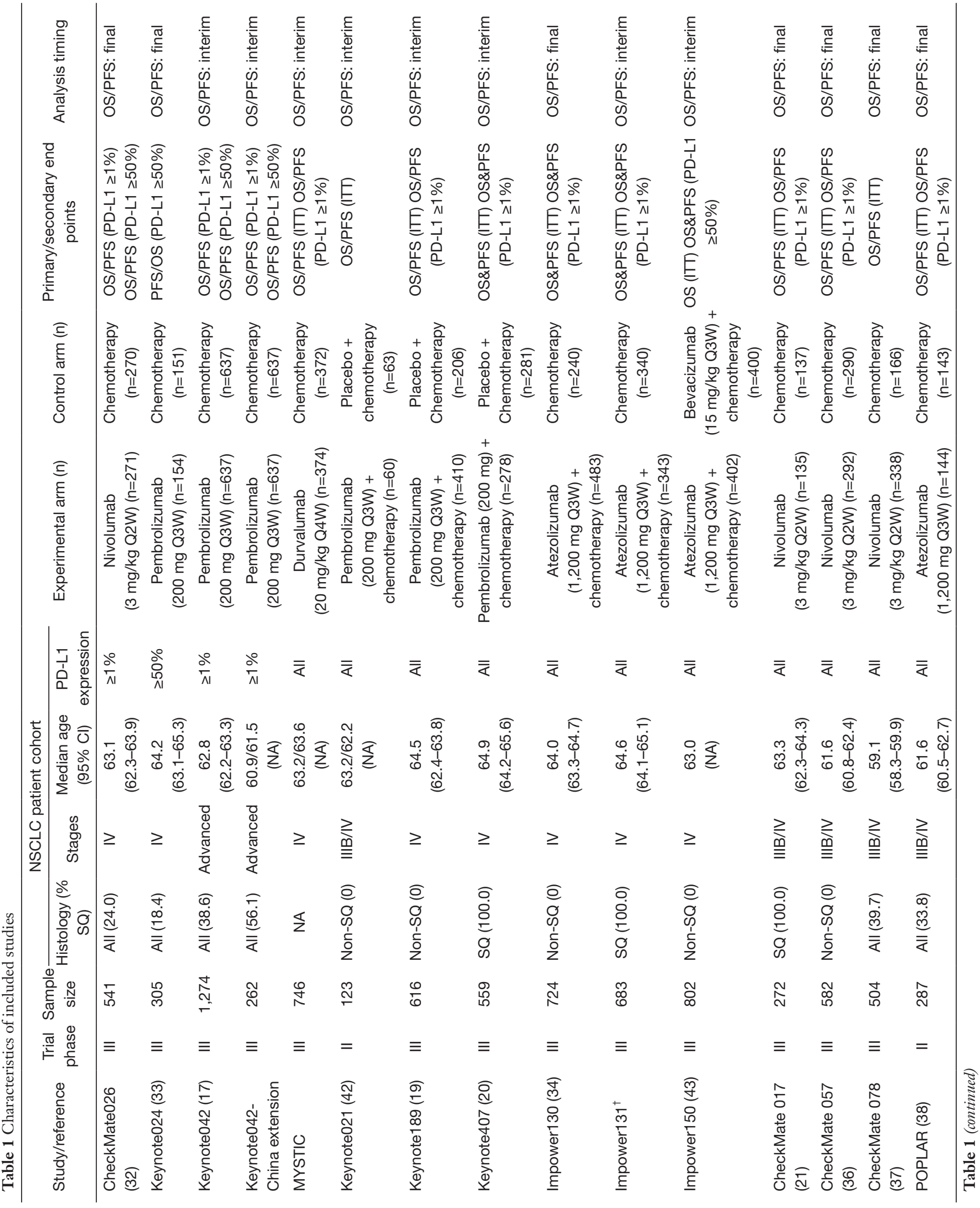



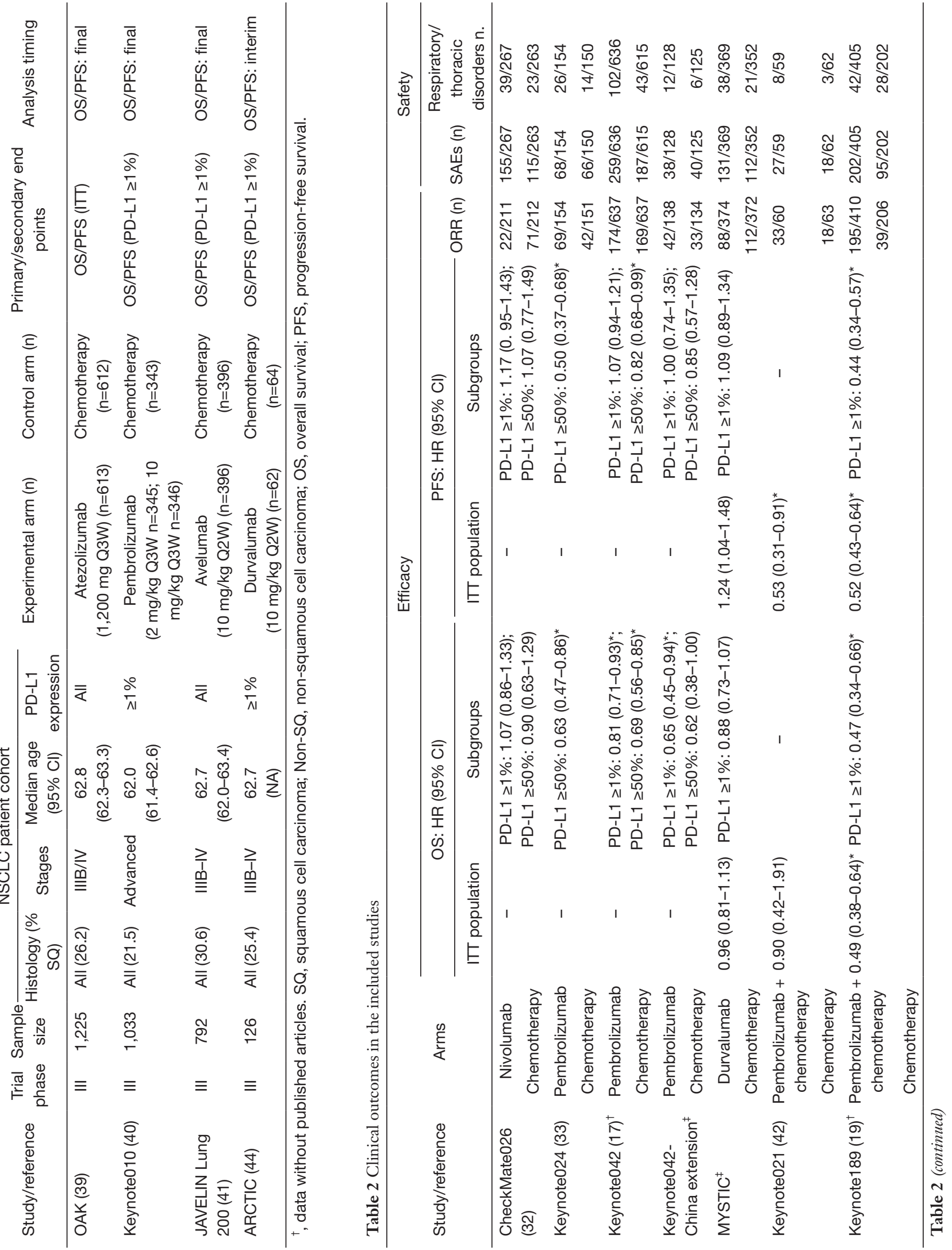


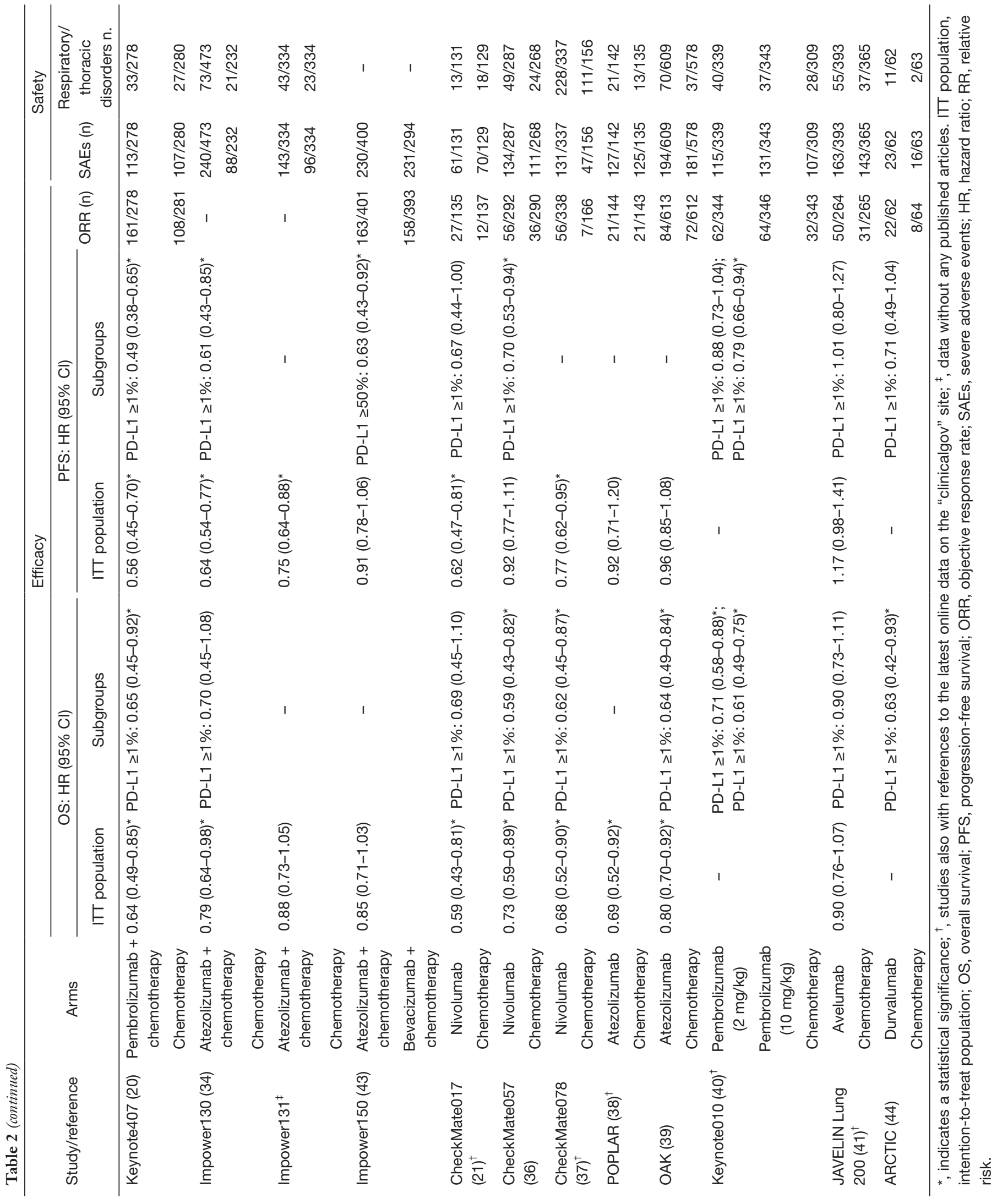




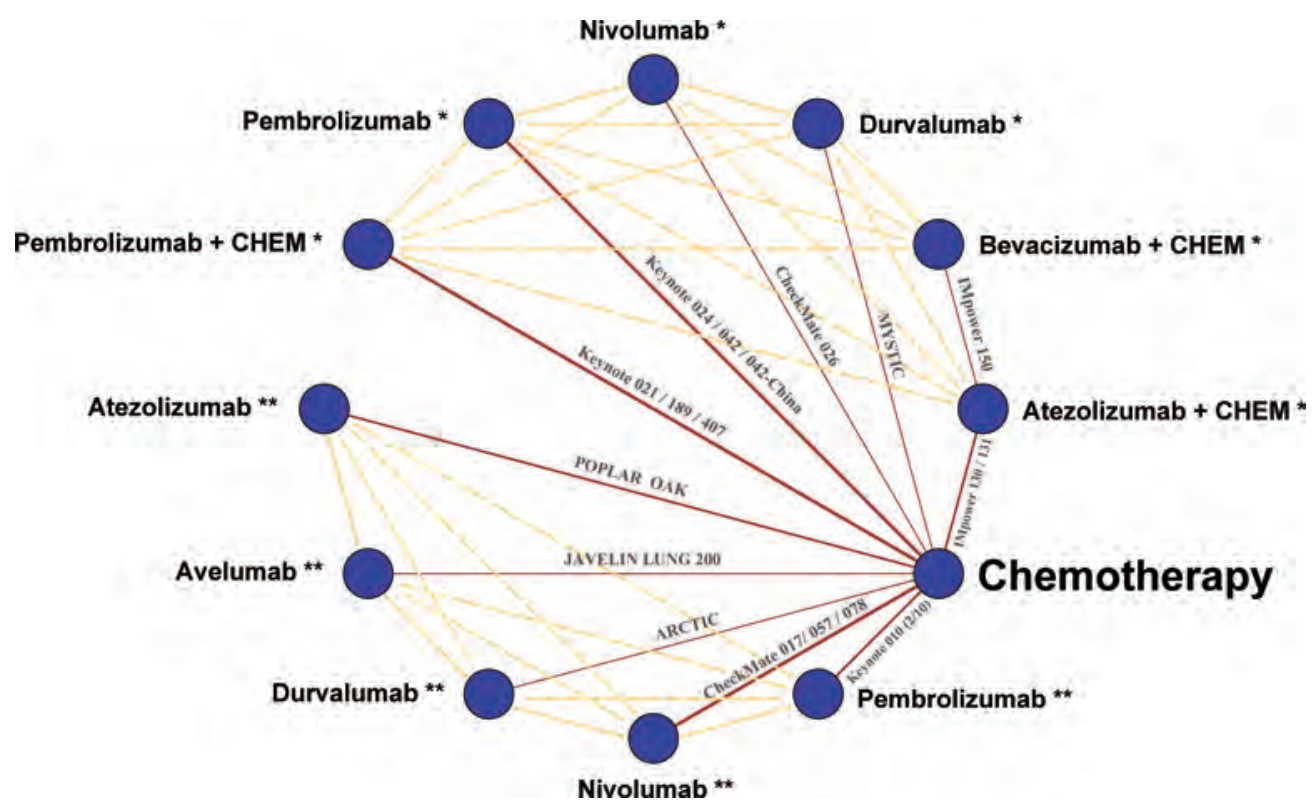

Figure 2 Network graph of trials assessing PD-1/L1 inhibitors in advanced NSCLC. *, in the first-line settings; **, in the second- or further-line settings. NSCLC, non-small cell lung cancer.

nivolumab was also of no significance either in OS or PFS (Table 3). Of note, heterogeneities between studies in the above NMAs were measured to be low or medium in terms of the $\mathrm{I}^{2}$ test.

In brief, the efficacy of the monotherapy supposed to be inferior to that of the combination therapy in the firstline settings according to the above results. For one thing, neither of durvalumab nor nivolumab monotherapy should be advised to patients because they even appeared to be weaker than chemotherapy when inhibiting the growth of tumors. For another, although it was relatively better than the durvalumab and nivolumab, pembrolizumab monotherapy was still not as effective as the combination therapy of pembrolizumab or atezolizumab, especially for patients harboring tumors that express a low or moderate status of PD-L1. However, these results are still needed to be further validated in future clinical trials.

\section{Second- or further-line settings}

Two populations, ITT as well as patients with PD-L1 TPS $\geq 1 \%$, were analyzed in these settings. Pooled analyses in the ITT patient population showed a significantly lower risk of death of PD-1/L1 inhibitors when compared to chemotherapy in OS [OS HR $\mathrm{HRoled}=0.77 ; 95 \%$ CI $(0.71,0.84)$; $\mathrm{I}^{2}=37 \%$ ] (Figure S4A). However, they showed no significance in reducing the risks of progression among pretreated patients in terms of PFS [PFS $\mathrm{HR}_{\text {pooled }}=0.92 ; 95 \% \mathrm{CI}(0.85$,
1.00); $\mathrm{I}^{2}=0 \%$ ] (Figure S4B) but showed significantly better ORRs indicated by the pooled RR [ORR RR $\mathrm{Rooled}_{\text {p }}=1.67 ; 95 \%$ CI (1.44, 1.93); $\mathrm{I}^{2}=56 \%$ ] (Figure S4C). Subgroups analyses among patients with PD-L1 TPS $\geq 1 \%$ showed similar results in OS [OS $\mathrm{HR}_{\text {pooled }}=0.69 ; 95 \% \mathrm{CI}(0.63,0.77) ; \mathrm{I}^{2}=35 \%$ ] (Figure S4D) and also PFS [PFS HR $\mathrm{Hooled}=0.82,95 \% \mathrm{CI}$ $\left.(0.75,0.91), \mathrm{I}^{2}=21 \%\right]$ (Figure $S 4 E$ ). Here, sensitivity analyses were conducted on PFS in the ITT population and OS among patients with PD-L1 TPS $\geq 1 \%$. Through omitting CheckMate017, a study with a relatively smaller sample size than the other studies of nivolumab and chemotherapy, the significance of advantage of anti-PD-1/L1 drugs in inhibiting tumor progression when compared with chemotherapy changed. The extent of benefits in OS that patients with PD-L1 TPS $\geq 1 \%$ could obtain from anti-PD-1/L1 drugs increased after the study related to durvalumab, ARCTIC, was excluded from the pooled analysis.

According to the ranking results from NMA, the top three regimens in terms of efficacy were nivolumab, atezolizumab, and avelumab in the ITT population. Nivolumab ranked as the best option for pretreated NSCLC patients, with probabilities of $77 \%$ in OS and $83 \%$ in PFS (Figure $4 A, B$ ). Its superiority to atezolizumab, the second-place inhibitor was significant both in PFS and ORR, but not in OS [PFS $\mathrm{HR}_{\text {indirect }}=0.84 ; 95 \% \mathrm{CI}(0.71$, $0.99) ; I^{2}=21 \%$; ORR $R_{\text {indirect }}=1.73 ; 95 \% \mathrm{CI}(1.16,2.58)$; 


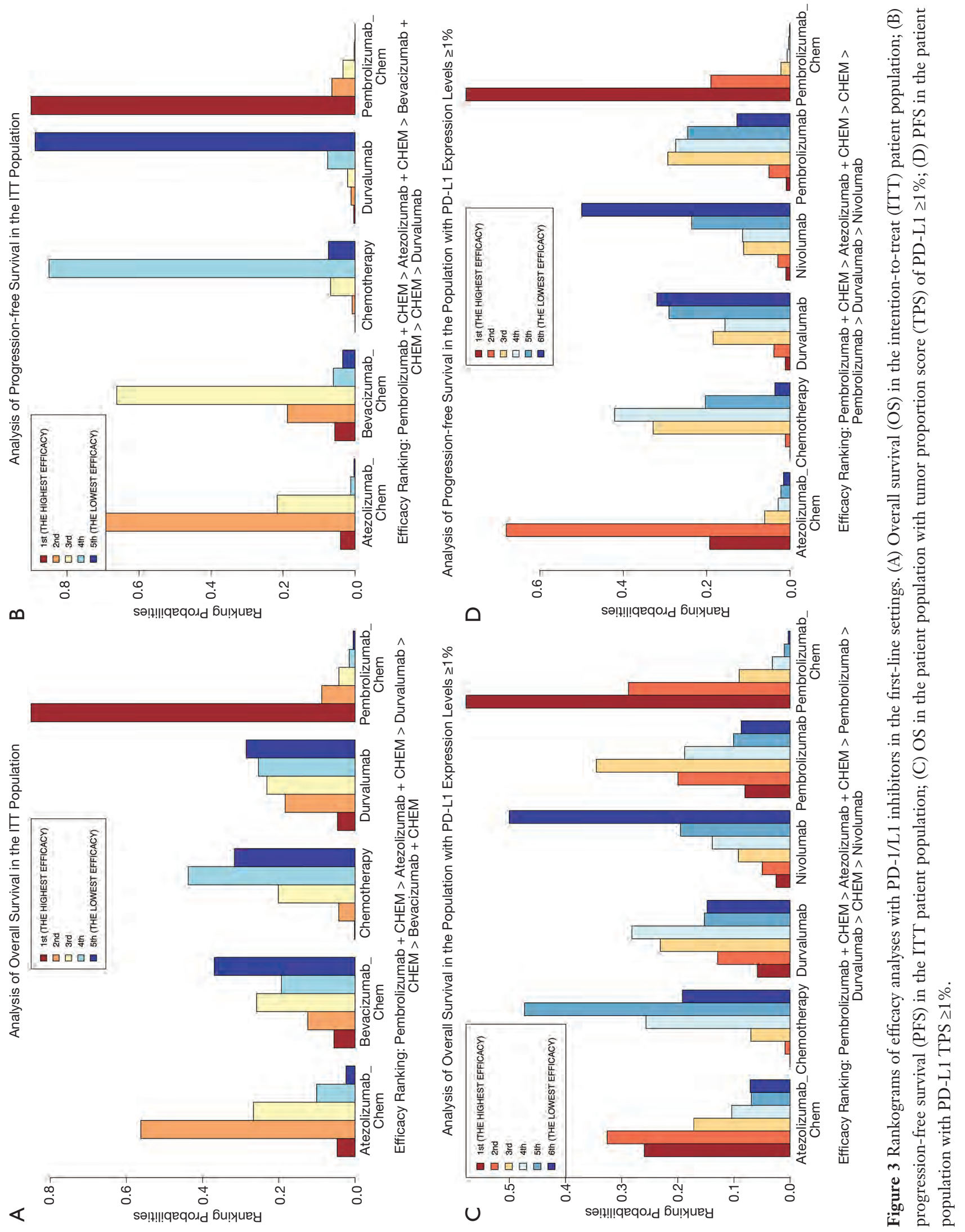


Table 3 Results of indirect comparisons in the first-line settings

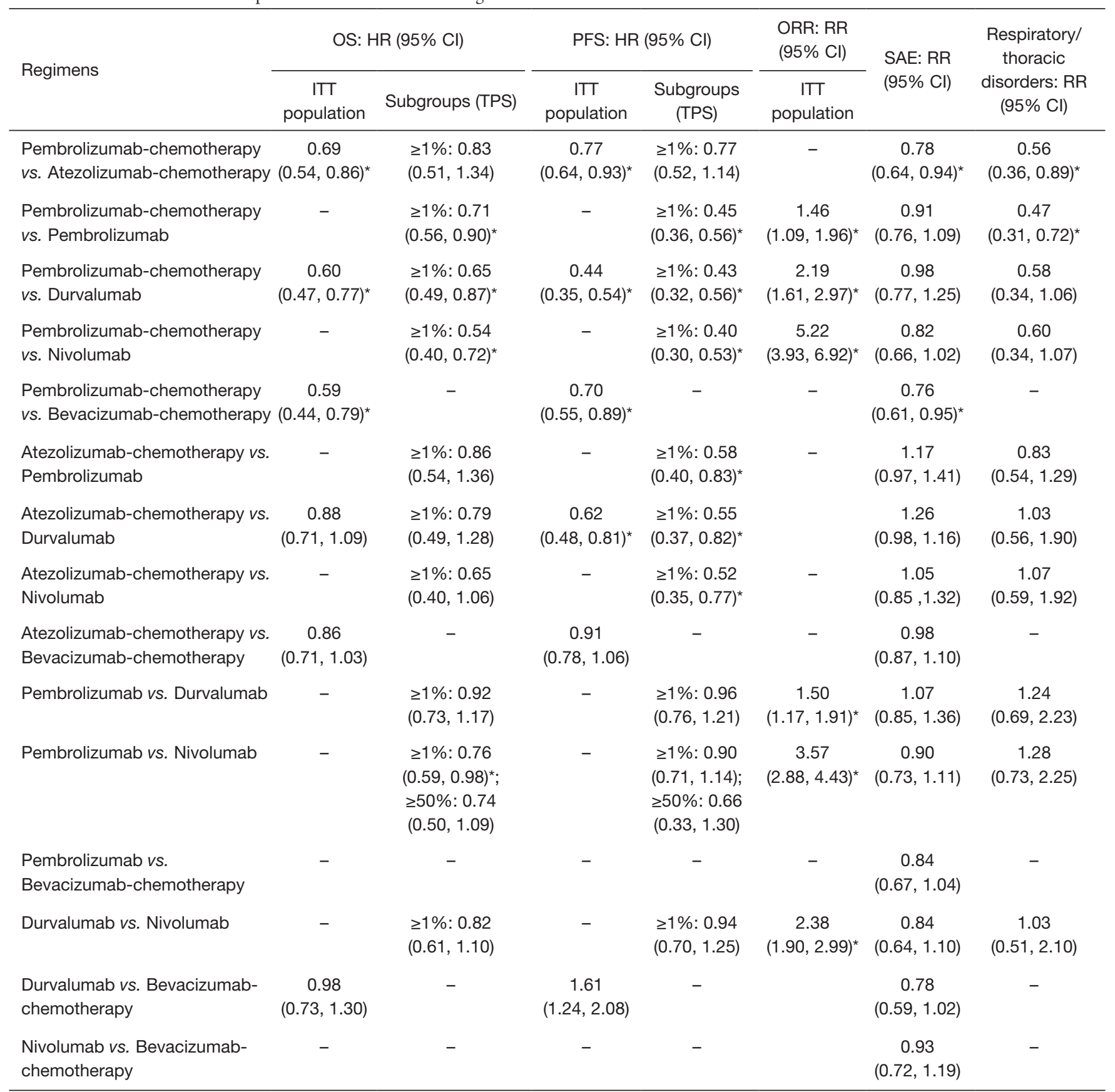

* indicates a statistical significance in each indirect comparison. ITT population, intention-to-treat population; TPS, tumor proportion score; OS, overall survival; PFS, progression-free survival; ORR, objective response rate; SAEs, severe adverse events; HR, hazard ratio; $\mathrm{RR}$, relative risk. 

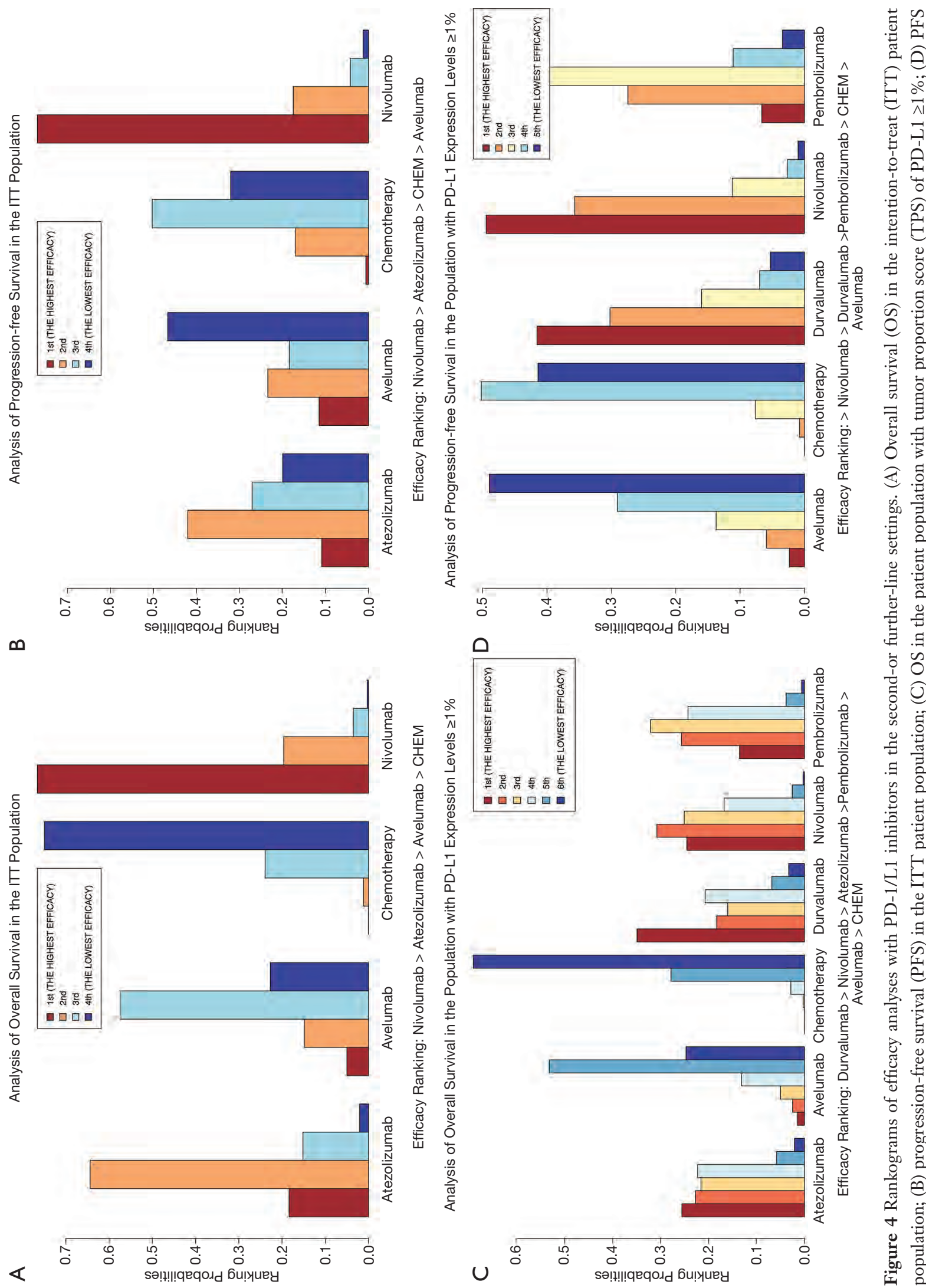

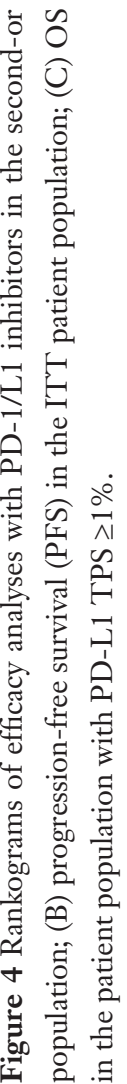


$\left.\mathrm{I}^{2}=15 \%\right]$. However, significance was shown in OS when comparing nivolumab with the third-rank avelumab [OS $\left.\mathrm{HR}_{\text {indirect }}=0.76 ; 95 \% \mathrm{CI}(0.60,0.95) ; \mathrm{I}^{2}=0.8 \%\right]$, but there was no significant difference between the second-rank agent, atezolizumab, and the third-rank avelumab either in terms of OS, PFS or ORR (Table 4).

In the subgroup NMA among patients with PD-L1 TPS $\geq 1 \%$, durvalumab, nivolumab, atezolizumab, and pembrolizumab supposed to be the top-best effective drugs for patients. Durvalumab ranked the best in terms of OS and ORR, with a probability of $35 \%$ and $55 \%$, respectively (Figure 4C,S5). Nivolumab was the best among all treatments with a probability of $47 \%$ in PFS (Figure 4D). Both of durvalumab and nivolumab were significantly superior to atezolizumab in ORR [ORR $\mathrm{RR}_{\text {indirect }}=2.52$; $95 \%$ CI $(1.16,5.47)$; ORR $\mathrm{RR}_{\text {indirect }}=1.73 ; 95 \% \mathrm{CI}(1.16$, $2.58) ; \mathrm{I}^{2}=15 \%$, but the superiority of durvalumab or nivolumab to pembrolizumab was insignificant either in terms of OS, PFS or ORR (Table 4). Of note, there was no significant difference between durvalumab and nivolumab either in terms of OS, PFS or ORR.

Atezolizumab ranked third in terms of OS in the population with PD-L1 TPS $\geq 1 \%$, and pembrolizumab took third place in terms of ORR (Figure 4C,S5). Because of the lacking data of atezolizumab, pembrolizumab also appeared to be the third-best effective agent with a probability of $51 \%$ in terms of PFS temporarily. However, other than the results in terms of ORR, which showed significant inferiority in efficacy for atezolizumab to pembrolizumab [ORR $\mathrm{RR}_{\text {indirect }}=0.58 ; 95 \% \mathrm{CI}(0.36,0.94)$; $\left.\mathrm{I}^{2}=15 \%\right]$, there was no significant difference between atezolizumab and pembrolizumab on their efficacy (Table 4). All of the heterogeneity between studies in the above NMAs in this setting was measured to be low or medium in terms of the I-square test.

\section{Drug safety \\ First-line settings}

Safety profiles were evaluated in the populations consisting of patients who had received more than one dose of treatment in each study. In the first-line settings, PD-1/ L1 inhibitors, either used as monotherapy or combined therapy, showed greater toxicity than chemotherapy alone, as indicated in the pooled RR with SAEs [SAE $\mathrm{RR}_{\text {pooled }}$ $\left.=1.23 ; 95 \% \mathrm{CI}(1.15,1.31) ; \mathrm{I}^{2}=48 \%\right]$ as well as respiratory and thoracic disorders [respiratory and thoracic disorders $\left.\mathrm{RR}_{\text {pooled }}=1.65 ; 95 \% \mathrm{CI}(1.41,1.93) ; \mathrm{I}^{2}=51 \%\right]$ (Figure S6A,B).

According to the NMAs, pembrolizumab combined chemotherapy was the best choice among all PD-1/L1 inhibitor therapies for the reduction of both incidences of SAEs and respiratory and thoracic disorders among the top three regimens (pembrolizumab combined with chemotherapy, atezolizumab combined with chemotherapy, and pembrolizumab monotherapy) in terms of efficacy in the first-line settings (Figure $5 A, B$ ). Its superiority to atezolizumab combined therapy was both significant in terms of reducing the incidence of SAEs and respiratory and thoracic disorders $\left[\mathrm{SAE} \mathrm{RR}_{\text {indirect }}=0.78 ; 95 \% \mathrm{CI}(0.64\right.$, $0.94) ; I^{2}=14 \%$; respiratory or thoracic disorders; $R R_{\text {indirect }}$ $=0.56 ; 95 \%$ CI $\left.(0.36,0.89) ; I^{2}=7 \%\right]$. Safety comparisons between pembrolizumab combined therapy and its monotherapy were also shown to be of significance in terms of respiratory and thoracic disorders [respiratory or thoracic disorders; $\mathrm{RR}_{\text {indirect }}=0.47 ; 95 \% \mathrm{CI}(0.31,0.72)$; $\left.\mathrm{I}^{2}=7 \%\right]$, but results were insignificant in terms of SAEs $\left[\mathrm{SAE} \mathrm{RR}_{\text {indirect }}=0.91 ; 95 \% \mathrm{CI}(0.76,1.09) ; \mathrm{I}^{2}=14 \%\right.$ ] (Table 3). Besides, pembrolizumab monotherapy tended to be safer than atezolizumab combined therapy in terms of SAEs, but atezolizumab showed greater benefits for patients in abating incidences of respiratory and thoracic disorders, however, with no significance both (Figure 5A,B; Table 3). All of the heterogeneity between studies in the above pair-wise analyses and NMAs was measured to be low or medium in terms of the I-square test.

\section{Second- or further-line settings}

As indicated in pooled RRs, PD-1/L1 inhibitors were found to be prone to increase the incidence of SAEs compared to chemotherapy [SAE $\mathrm{RR}_{\text {pooled }}=1.06 ; 95 \% \mathrm{CI}(0.99,1.15)$; $\mathrm{I}^{2}=8 \%$ ] (Figure S6C), which was similar to the incidence of respiratory and thoracic disorders [respiratory and thoracic disorders $\mathrm{RR}_{\text {pooled }}=1.48$; $95 \%$ CI $(1.24,1.76)$; $\left.\mathrm{I}^{2}=37 \%\right]$ (Figure S6D). Sensitivity analyses were conducted based on the above analyses. By excluding POPLAR, a study with a relatively smaller sample size than OAK, the extent of hazards that patients would suffer from SAEs slightly increased. Besides, patients were much more likely to develop respiratory and thoracic disorders after using PD-1/L1 inhibitors than chemotherapy after omitting CheckMate078, which had included a relatively young patient population.

According to the NMAs, durvalumab was the most toxic agent for patients no matter in terms of SAEs or respiratory and thoracic disorders among the top regimens (durvalumab, nivolumab, atezolizumab, and pembrolizumab) in terms of efficacy in the second- or further-line settings. In contrast, nivolumab ranked as the safest inhibitor in reducing 


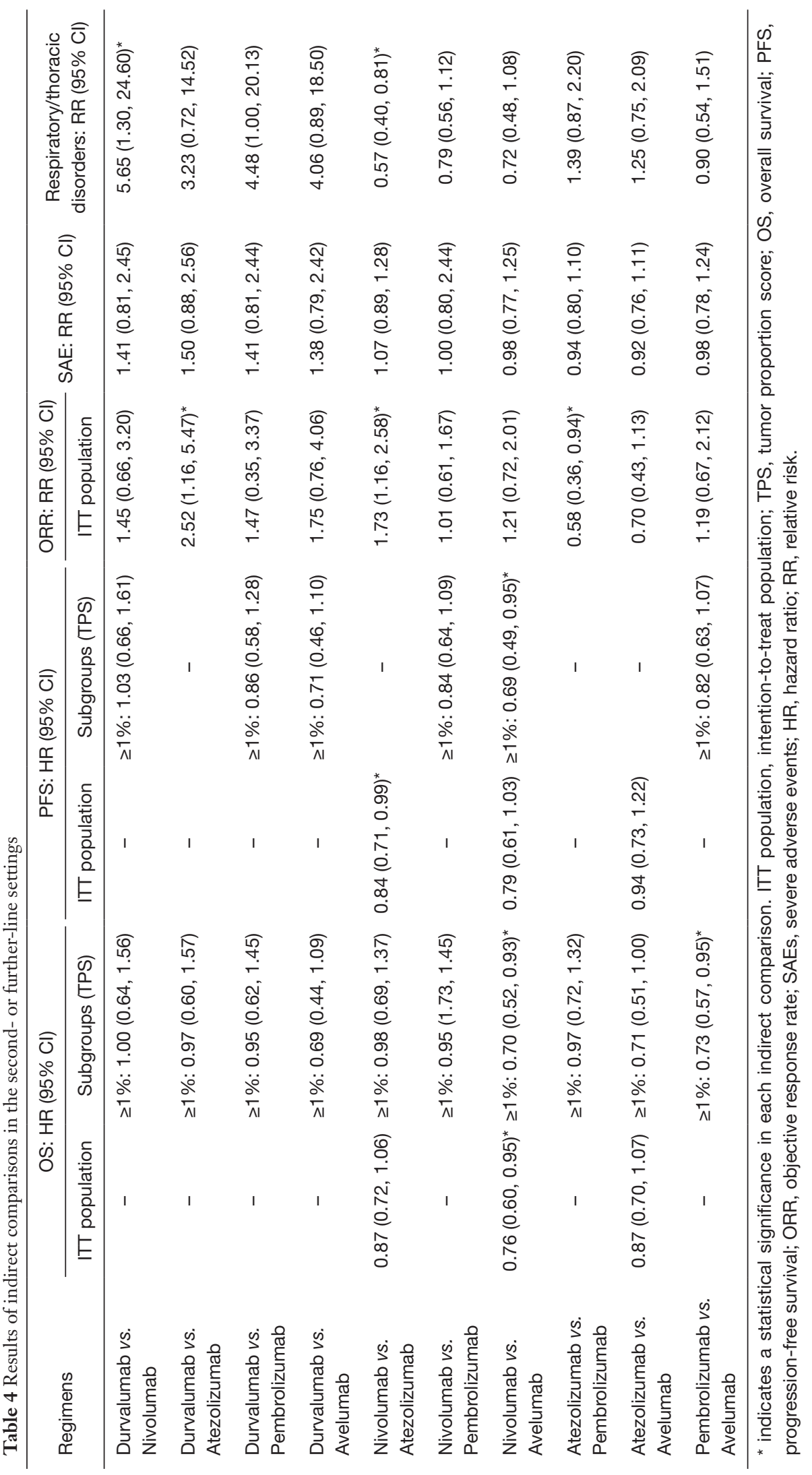



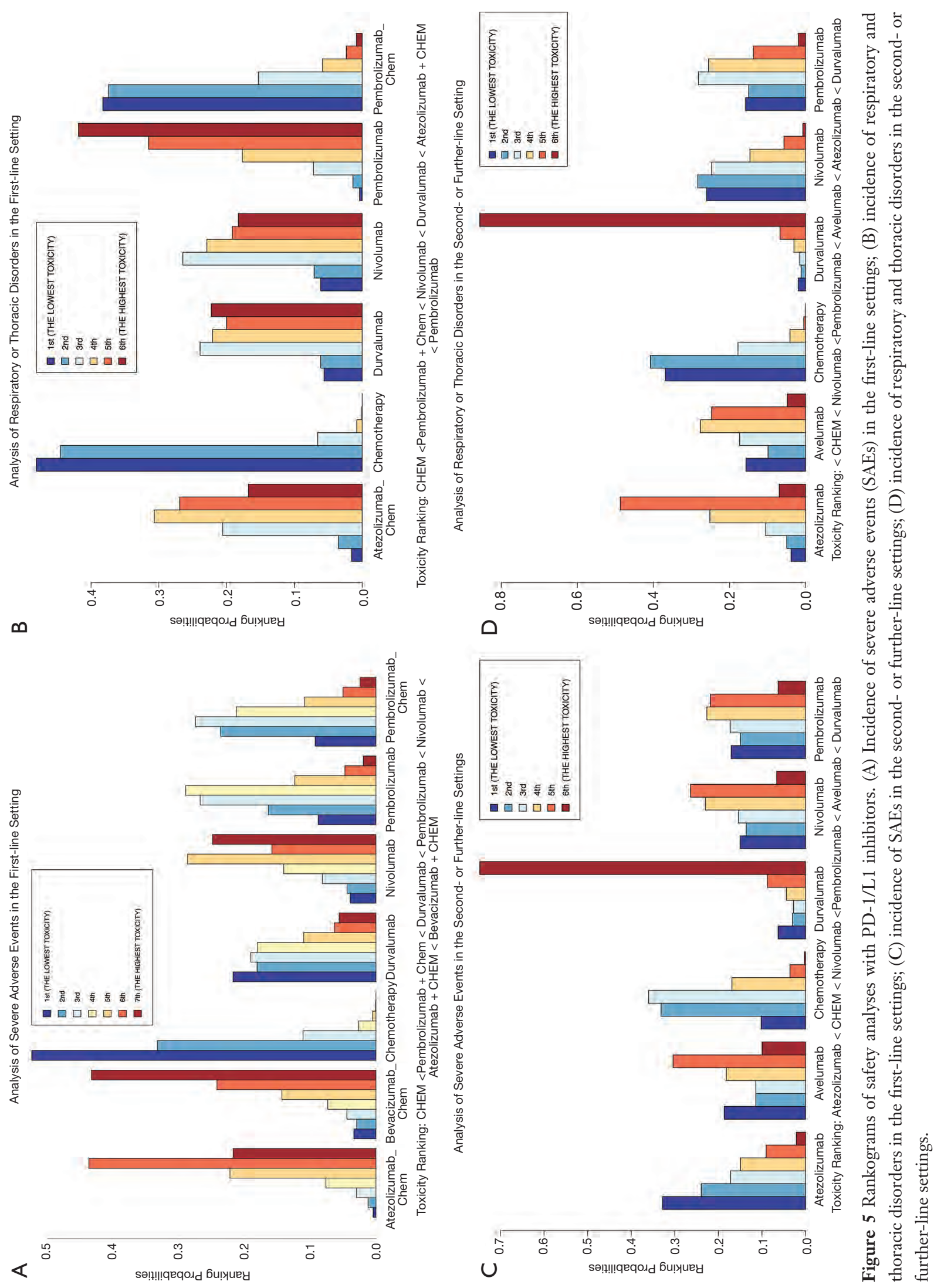
the incidence of respiratory and thoracic disorders, and atezolizumab seemed to be the safest agent in terms of SAEs (Figure 5C,D). Safety comparisons between nivolumab and atezolizumab were shown to be significant in terms of respiratory and thoracic disorders [respiratory or thoracic disorders; $\left.\mathrm{RR}_{\text {indirect }}=0.57 ; 95 \% \mathrm{CI}(0.40,0.81) ; \mathrm{I}^{2}=15 \%\right]$, but results were insignificant in terms of SAEs (Table 4). Besides, atezolizumab tended to be safer than pembrolizumab in terms of SAEs, but pembrolizumab showed greater benefits for patients in abating incidences of respiratory and thoracic disorders, however, with no significance in both of these results (Figure 5C,D; Table 4). All of the heterogeneity between studies in the above NMAs was measured to be low in terms of the I-square test.

\section{Discussion}

Immunotherapy represents a novel approach in management for patients with NSCLC, in which several ICIs are currently being investigated (45). One of the most studied immune checkpoints is PD-1 together with its ligand PD-L1. PD-1 is transcriptionally induced on activated T cells, and PD-L1 expression is found in multiple tumors including $20-65 \%$ of NSCLC (46). To date, three common ICIs, including nivolumab, pembrolizumab, and atezolizumab, have been approved for treatments for lung cancer $(36,47,48)$, while durvalumab and avelumab are still in their clinical development stages. The approvals were based on the improvement in OS and better safety profiles comparing anti-PD-1/L1 agents to other regimens such as chemotherapy, which was reported in RCTs. However, there scarcely exists head-to-head trial evidence or an authoritative guideline for clinicians to determine an optimal agent for diverse patients with NSCLC. Given this condition, NMA could be used to compare these agents by combining direct comparisons with indirect ones using results from the RCTs in a network simultaneously $(49,50)$.

According to results from efficacy NMA and indirect comparisons in the first-line settings, pembrolizumab combined therapy seemed to be the best option in terms of efficacy for advanced NSCLC ITT patients and those with PD-L1 TPS $\geq 1 \%$. Pembrolizumab was the optimal choice for those patients whose tumors harbor PD-L1 TPS $\geq 50 \%$. Safety NMAs in this setting showed decreasing risks of SAEs for pembrolizumab combined therapy as well as its monotherapy compared to their counterparts. Combining principal outcomes of efficacy and safety, including OS and PFS as well as the incidence of SAEs, we concluded that the addition of pembrolizumab to chemotherapy was an appropriate choice for patients with positive tumor expressions of PD-L1 (TPS $\geq 1 \%$ ). Patients with high expressions of PD-L1 (TPS $\geq 50 \%$ ) could adopt pembrolizumab alone for better outcomes in clinical practice. In order to compare the advantages and disadvantages of each regimens more comprehensively, efficacy-and-safety-inclusive advantages of each regimen from our analyses were presented with stacked bar charts, in which averages of the first- and second-best ranking probabilities in terms of efficacy (OS and PFS) and safety (SAEs) were accumulated together (Figures 6A,B,S7).

Given results from NMAs in the second- or further-line settings, nivolumab and durvalumab became the most two preferred inhibitors in terms of drug efficacy for advanced NSCLC patients, regardless of the PD-L1 status of their tumors. Atezolizumab and pembrolizumab were alternative options because the results of indirect comparisons between nivolumab and them each were not all significant. Considering drug safety profiles, atezolizumab was the most tolerable inhibitor for advanced NSCLC patients in terms of SAEs, whereas nivolumab was the safest agent in terms of respiratory and thoracic disorders, and also it was the second-safest drug in terms of SAEs. Pembrolizumab was less safe than nivolumab either in terms of SAEs or respiratory and thoracic disorders, but it could bring less incidence of respiratory and thoracic disorders than atezolizumab to advanced NSCLC patients. In contrast, durvalumab was surprisingly the most toxic anti-PD-1/L1 drug in terms of SAEs as well as respiratory and thoracic disorders. Combining principal outcomes of efficacy and safety, including OS and PFS or ORR as well as the incidence of SAEs, nivolumab was the most appropriate PD-1/L1 inhibitor for patients who had failed in previous chemotherapies, regardless of the expression of PD-L1 of their tumors. Durvalumab could also be recommended for these patients only if they had relatively good performances. Atezolizumab was an alternative for patients with a generally poor clinical condition without severe pulmonary symptoms. Stacked bar charts incorporating accumulated averages of the first- and second-best ranking probabilities in terms of efficacy (OS and PFS or ORR) and safety (SAEs) are displayed to present the comprehensive advantages of each regimen in these settings (Figure 6C,D).

Our NMA is not the first such analysis, but we included avelumab and durvalumab for the first time, and we also incorporated the latest updated data of all included studies. To the best of our knowledge, our study is the 

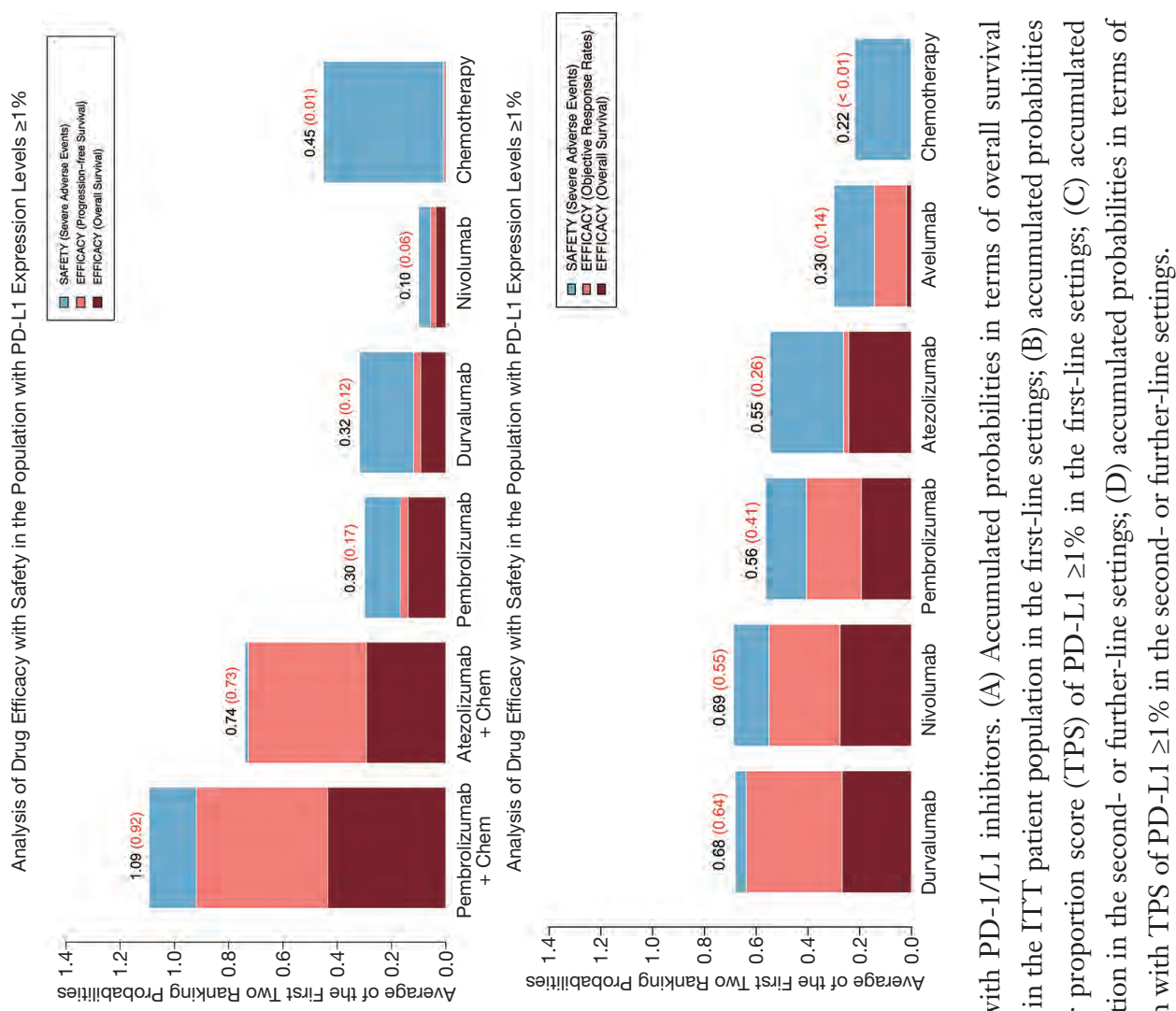

m

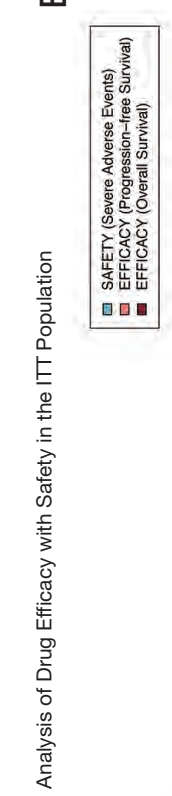

0
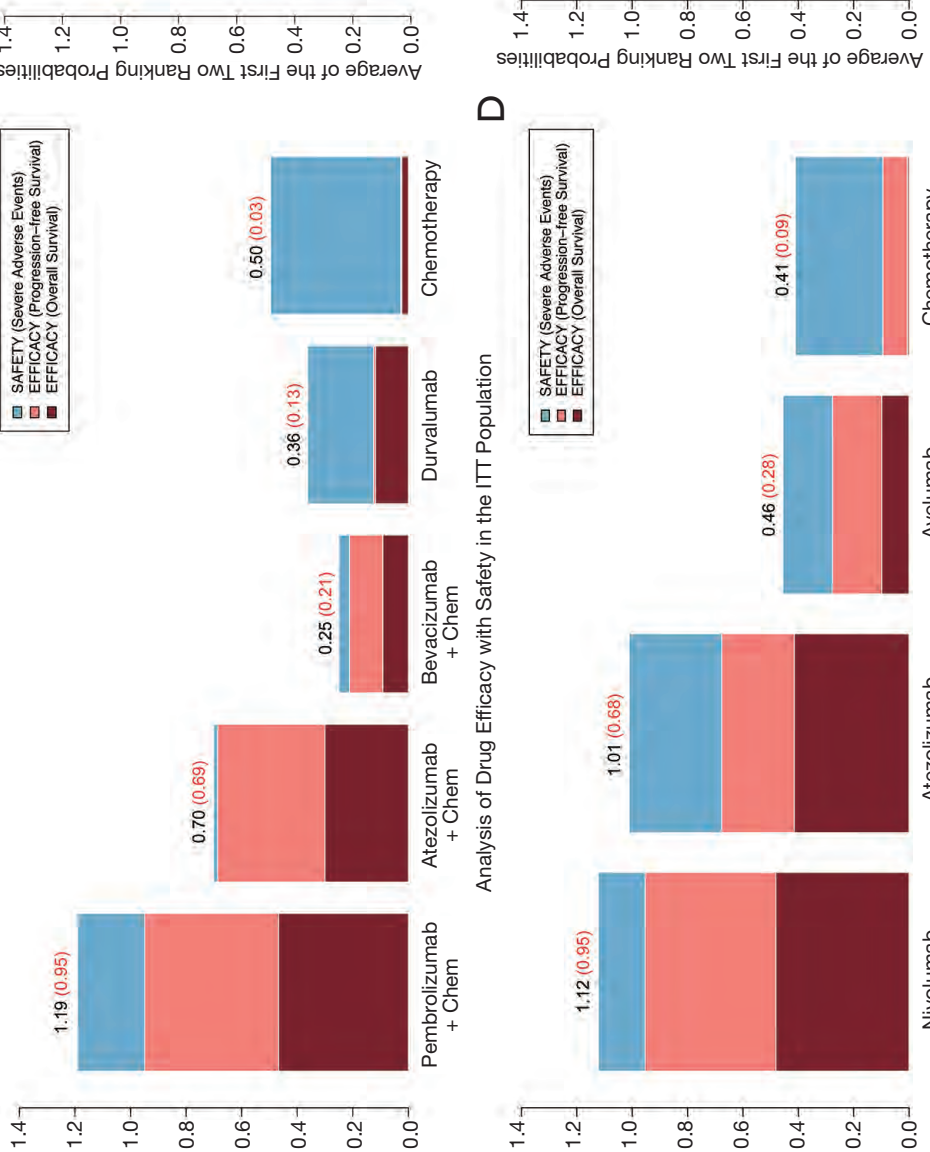

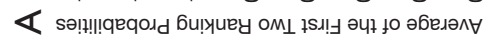
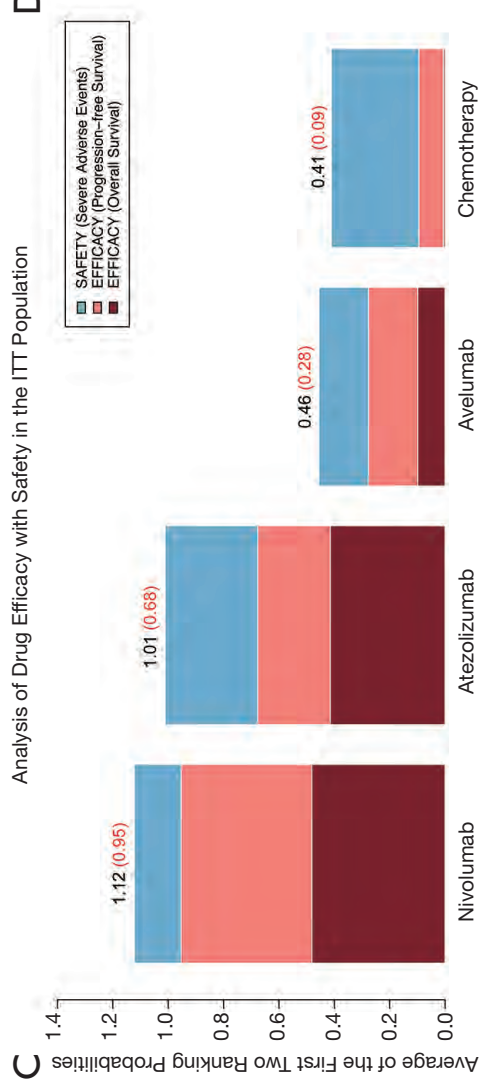

E.

它

声点

की

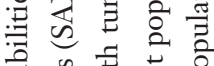

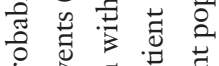

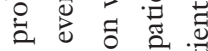

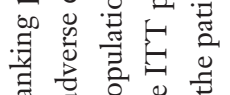
जٓ

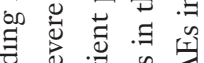

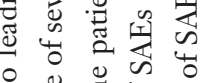

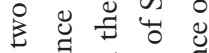

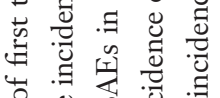

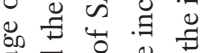

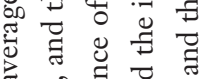

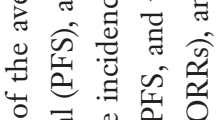

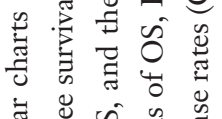

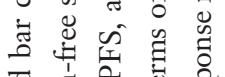
चु 芯

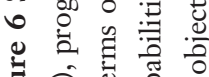

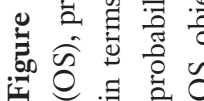


most comprehensive analysis involving all treating lines confined with chemotherapeutic drugs and PD-1/L1 inhibitors. Despite these strengths, there have been several controversies. Three studies associated with NMA had been conducted to figure out the best PD-1/L1 inhibitor for advanced NSCLC patients in the first-line settings (51-53). Our results were generally consistent with the previous studies, which had all indicated the combination of pembrolizumab to be better than other combined therapies or chemotherapies for patients with relatively high expression of PD-L1. However, atezolizumab and chemotherapy had also been reported to be appropriate for this subset of patients in one of these studies (52). Controversy might have resulted from the insufficient data of atezolizumab in our study, which was partly due to the exclusion of study Impower132, whose results had only been reported at a conference, with interim outcomes. In this case, we further analyzed the efficacy results of atezolizumab combined therapy in our study with the studies Impower 130 and 131. We found that although the front-ranking probabilities of atezolizumab combined therapy were relatively high in patients with positive PDL1 expression (TPS $\geq 1 \%$ ), it was still significantly less beneficial for patients than pembrolizumab combined therapy according to the results from indirect comparisons. Safety analyses reported that this combined regimen seemed to be significantly less safe than pembrolizumab combined therapy. We, therefore, believe that the combination of atezolizumab and chemotherapy is indeed inferior to the pembrolizumab combined therapy when considering efficacy and safety together, but the results still need validations by RCTs in the future. Besides, five studies had been performed to compare PD-1/L1 inhibitors in the subsequent settings (54-58). Drug efficacy was the primary outcomes reported at the end of these studies while only a few studies focused on drug safety. Nivolumab was recommended consistently by almost all of these five studies, four of which also suggested pembrolizumab with no significant difference reported between these two regimens (54-56,58). The comparison on drug safety between these two drugs had only been analyzed by one study, which had reported that pembrolizumab had a higher incidence of adverse effects than nivolumab, which was consistent with our results (54). Atezolizumab had been recommended by the authors in three of these previous studies (55-57), which was also consistent with our study.

There were still some limitations in our study. First, there have been two existing parameters for the testing of PD-L1 expression cut-offs, tumor cell and tumorinfiltrating immune cell staining, and the cut-offs also varied in different studies. In our study, the included trials were shown to use one or both of these two methods, whereas in several trials, researchers had not completely clarified the parameters that had been measured. Thus, caution must be used when interpreting subgroup results. Second, nine included studies only reported interim results in OS. Because immunotherapy requires a longer followup to manifest its benefits on patient survival, this limitation might lead to effects on the final pooled and indirect results. Hence, it is a necessity to perform further analyses in the future. Third, two dosages of drugs had been evaluated in the included study Keynote 010, involving 2 and $10 \mathrm{mg} / \mathrm{kg}$, which could lead to a relative overestimation of the drugrelated toxicity as well as efficacy in the second- or furtherline settings, because the dosage of $2 \mathrm{mg} / \mathrm{kg}$ is currently approved for clinical use. Thus, the efficacy and safety profiles of pembrolizumab compared to other inhibitors need to be validated by further research. Fourth, subgroup analyses related to the histological types of NSCLC on the regimens were not carried out in this work, because outcomes of patients with different histological types were hardly reported in the included studies according to reported details of each included study. Moreover, the pooled and indirect results associated with atezolizumab combined therapy in the first-line settings among patients with PD-L1 TPS $\geq 1 \%$ only involved those with squamous histology, leading to the only potential heterogeneity and non-transitive result in our study. Thus, it might be an interesting issue for researchers to analyze that whether the survival of NSCLC patients with various histological types differs after treated with PD-1/L1 inhibitors. Fifth, SAEs which were defined as interferes with normal life functions or events requiring extending inpatient hospitalization were subjective and might have contributed to the observer bias because the trials were all open-label. In addition, the degree of classifications in respiratory and thoracic disorders varied between studies; for example, categories of disorders in larger clinical studies with more patients involved appeared to be more detailed. Our results were therefore suggestive but not conclusive, and caution is recommended in the interpreting. Head-to-head clinical trials comparing these emerging $\mathrm{PD}-1 / \mathrm{L} 1$ inhibitors are still a necessity to reduce the limitations from indirect comparisons, to identify the optimal treatments for advanced NSCLC. Last but not least, we did not include any anti-CTLA-4 drugs in our analyses. Since CTLA-4 is another pivotal 
checkpoint which is practical for anticancer therapies, and the combination of CTLA-4 and PD- 1 inhibitors has been suggested to have synergistic effect on the activation of antitumor immune response and to increase the response rates in patients, multiple studies have investigated the efficacy of anti-PD-1/L1 combined with anti-CTLA-4 antibodies in lung cancer (59-62). Thus, the differences in efficacy and safety between this combined therapy and that analyzed by us should also be assessed by further studies. Besides, other combination therapies that contain more than one checkpoint inhibitors are also worthy of assessment in the field of managements for advanced NSCLC patients.

\section{Conclusions}

The drug efficacy of regimens including PD-1/L1 inhibitors is superior to chemotherapy for NSCLC, regardless of the therapeutic line used. The monotherapy of these inhibitors seemed to be inferior to that of the combination therapy. When using PD-1/L1 inhibitors in the clinical practice, PD-L1 expression status, physical conditions, and whether they have received systemic therapy should all be considered for patients. Pembrolizumab in combination with chemotherapeutic drugs is appropriate for patients with a low expression of PD-L1, while patients with a high expression of PD-L1 can also choose pembrolizumab alone for better clinical outcomes. In the second- or furtherline settings, nivolumab is an optimal choice for patients with advanced NSCLC. The novel inhibitor, durvalumab, can only be tried by patients with relatively good physical performances for better clinical outcomes. Atezolizumab would be an alternative for patients with poor general conditions, but it should not be suggested when thoracic disorders have already occurred in them. However, our results need to be further validated in future clinical trials.

\section{Acknowledgments}

The authors thank International Science Editing Co. (http:// www.internationalscienceediting.com) for editing this manuscript.

Funding: This work was supported by the National Natural Science Foundation of China (Grant Nos. 81672268, 81572295; www.nsfc.gov.cn).

\section{Footnote}

Reporting Checklist: The authors have completed the
PRISMA reporting checklist. Available at http://dx.doi. org/10.21037/tlcr-20-192

Conflicts of Interest: All authors have completed the ICMJE uniform disclosure form (available at http://dx.doi. org/10.21037/tlcr-20-192). The authors have no conflicts of interest to declare.

Ethical Statement: The authors are accountable for all aspects of the work in ensuring that questions related to the accuracy or integrity of any part of the work are appropriately investigated and resolved. Ethical approval was exempted by the Institutional Review Committee of Zhongshan Hospital, Fudan University, Shanghai, China (approval number: B2019-232R).

Open Access Statement: This is an Open Access article distributed in accordance with the Creative Commons Attribution-NonCommercial-NoDerivs 4.0 International License (CC BY-NC-ND 4.0), which permits the noncommercial replication and distribution of the article with the strict proviso that no changes or edits are made and the original work is properly cited (including links to both the formal publication through the relevant DOI and the license). See: https://creativecommons.org/licenses/by-nc-nd/4.0/.

\section{References}

1. Siegel RL, Miller KD, Jemal A. Cancer statistics, 2020. CA Cancer J Clin 2020;70:7-30.

2. Govindan R, Page N, Morgensztern D, et al. Changing epidemiology of small-cell lung cancer in the United States over the last 30 years: analysis of the surveillance, epidemiologic, and end results database. J Clin Oncol 2006;24:4539-44.

3. Li T, Ma W, Tian EC. Ensartinib (X-396): what does it add for patients with ALK-rearranged NSCLC. Chin Clin Oncol 2019;8:S4.

4. Yoneyama R, Saji H, Kato Y, et al. Clinicopathological characteristics and treatment strategies for young lung cancer patients. Ann Transl Med 2019;7:100.

5. Mok T, Yang JJ, Lam KC. Treating patients with EGFRsensitizing mutations: first line or second line--is there a difference? J Clin Oncol 2013;31:1081-8.

6. Velez MA, Burns TF. Is the game over for PD-1 inhibitors in EGFR mutant non-small cell lung cancer? Transl Lung Cancer Res 2019;8:S339-42.

7. Paz-Ares LG, de Marinis F, Dediu M, et al. 
PARAMOUNT: Final overall survival results of the phase III study of maintenance pemetrexed versus placebo immediately after induction treatment with pemetrexed plus cisplatin for advanced nonsquamous non-small-cell lung cancer. J Clin Oncol 2013;31:2895-902.

8. Hanna N, Shepherd FA, Fossella FV, et al. Randomized phase III trial of pemetrexed versus docetaxel in patients with non-small-cell lung cancer previously treated with chemotherapy. J Clin Oncol 2004;22:1589-97.

9. Travert C, Tomasini P, Jeanson A, et al. Firstline pembrolizumab in programmed death ligand 1 positive non-small cell lung cancer. Transl Cancer Res 2019;8:2514-6.

10. Yan Y, Zeng S, Wang X, et al. A Machine Learning Algorithm for Predicting Therapeutic Response to Anti-PD1. Technol Cancer Res Treat 2019;18:1533033819875766.

11. Iwai Y, Ishida M, Tanaka Y, et al. Involvement of PD-L1 on tumor cells in the escape from host immune system and tumor immunotherapy by PD-L1 blockade. Proc Natl Acad Sci U S A 2002;99:12293-7.

12. Gettinger SN, Horn L, Gandhi L, et al. Overall Survival and Long-Term Safety of Nivolumab (Anti-Programmed Death 1 Antibody, BMS-936558, ONO-4538) in patients with previously treated advanced non-small-cell lung cancer. J Clin Oncol 2015;33:2004-12.

13. Gettinger S, Rizvi NA, Chow LQ, et al. Nivolumab Monotherapy for First-Line Treatment of Advanced NonSmall-Cell Lung Cancer. J Clin Oncol 2016;34:2980-7.

14. Garon EB, Hellmann MD, Rizvi NA, et al. Five-Year Overall Survival for Patients With Advanced Non-SmallCell Lung Cancer Treated With Pembrolizumab: Results From the Phase I KEYNOTE-001 Study. J Clin Oncol 2019;37:2518-27.

15. Garassino MC, Cho BC, Kim JH, et al. Durvalumab as third-line or later treatment for advanced non-small-cell lung cancer (ATLANTIC): an open-label, single-arm, phase 2 study. Lancet Oncol 2018;19:521-36.

16. Gulley JL, Rajan A, Spigel DR, et al. Avelumab for patients with previously treated metastatic or recurrent non-smallcell lung cancer (JAVELIN Solid Tumor): dose-expansion cohort of a multicentre, open-label, phase $1 \mathrm{~b}$ trial. Lancet Oncol 2017;18:599-610.

17. Mok TSK, Wu YL, Kudaba I, et al. Pembrolizumab versus chemotherapy for previously untreated, PD-L1expressing, locally advanced or metastatic non-small-cell lung cancer (KEYNOTE-042): a randomised, open-label, controlled, phase 3 trial. Lancet 2019;393:1819-30.
18. Reck M, Rodríguez-Abreu D, Robinson AG, et al. Pembrolizumab versus chemotherapy for PD-L1-positive non-small-cell lung cancer. N Engl J Med 2016;375:1823-33.

19. Gandhi L, Rodríguez-Abreu D, Gadgeel S, et al. Pembrolizumab plus chemotherapy in metastatic nonsmall-cell lung cancer. N Engl J Med 2018;378:2078-92.

20. Paz-Ares L, Luft A, Vicente D, et al. Pembrolizumab plus chemotherapy for squamous non-small-cell lung cancer. $\mathrm{N}$ Engl J Med 2018;379:2040-51.

21. Vokes EE, Ready N, Felip E, et al. Nivolumab versus docetaxel in previously treated advanced non-small-cell lung cancer (CheckMate 017 and CheckMate 057): 3-year update and outcomes in patients with liver metastases. Ann Oncol 2018;29:959-65.

22. Wan N, Ji B, Li J, et al. A pooled meta-analysis of PD-1/L1 inhibitors incorporation therapy for advanced non-small cell lung cancer. Onco Targets Ther 2019;12:4955-73.

23. Peng M, Li X, Lei G, et al. The efficacy and safety of immune checkpoint inhibitor combination therapy in lung cancer: a systematic review and meta-analysis. Onco Targets Ther 2018;11:7369-83.

24. Nishijima TF, Shachar SS, Nyrop KA, et al. Safety and Tolerability of PD-1/PD-L1 Inhibitors Compared with Chemotherapy in Patients with Advanced Cancer: A MetaAnalysis. Oncologist 2017;22:470-9.

25. Nishino M, Giobbie-Hurder A, Hatabu H, et al. Incidence of programmed cell death 1 inhibitor-related pneumonitis in patients with advanced cancer: a systematic review and meta-analysis. JAMA Oncol 2016;2:1607-16.

26. Passiglia F, Galvano A, Rizzo S, et al. Looking for the best immune-checkpoint inhibitor in pre-treated NSCLC patients: An indirect comparison between nivolumab, pembrolizumab and atezolizumab. Int J Cancer 2018;142:1277-84.

27. Guyot P, Ades AE, Ouwens MJ, et al. Enhanced secondary analysis of survival data: reconstructing the data from published Kaplan-Meier survival curves. BMC Med Res Methodol 2012;12:9.

28. Parmar MK, Torri V, Stewart L. Extracting summary statistics to perform meta-analyses of the published literature for survival endpoints. Stat Med 1998;17:2815-34.

29. Higgins JP, Altman DG, Gotzsche PC, et al. The Cochrane Collaboration's tool for assessing risk of bias in randomised trials. BMJ 2011;343:d5928.

30. IntHout J, Ioannidis JP, Borm GF. The Hartung-KnappSidik-Jonkman method for random effects meta-analysis is straightforward and considerably outperforms the standard DerSimonian-Laird method. BMC Med Res Methodol 
2014;14:25.

31. Leonard T, Duffy JC. A Bayesian fixed effects analysis of the Mantel-Haenszel model applied to meta-analysis. Stat Med 2002;21:2295-312.

32. Carbone DP, Reck M, Paz-Ares L, et al. First-Line nivolumab in stage IV or recurrent non-small-cell lung cancer. N Engl J Med 2017;376:2415-26.

33. Reck M, Rodríguez-Abreu D, Robinson AG, et al. Updated Analysis of KEYNOTE-024: Pembrolizumab Versus Platinum-Based Chemotherapy for Advanced NonSmall-Cell Lung Cancer With PD-L1 Tumor Proportion Score of 50\% or Greater. J Clin Oncol 2019;37:537-46.

34. West H, McCleod M, Hussein M, et al. Atezolizumab in combination with carboplatin plus nab-paclitaxel chemotherapy compared with chemotherapy alone as firstline treatment for metastatic non-squamous non-smallcell lung cancer (IMpower130): a multicentre, randomised, open-label, phase 3 trial. Lancet Oncol 2019;20:924-37.

35. Brahmer J, Reckamp KL, Baas $P$, et al. Nivolumab versus docetaxel in advanced squamous-cell non-small-cell lung cancer. N Engl J Med 2015;373:123-35.

36. Kazandjian D, Suzman DL, Blumenthal G, et al. FDA Approval summary: nivolumab for the treatment of metastatic non-small cell lung cancer with progression on or after platinum-based chemotherapy. Oncologist 2016;21:634-42.

37. Wu YL, Lu S, Cheng Y, et al. Nivolumab Versus Docetaxel in a Predominantly Chinese Patient Population With Previously Treated Advanced NSCLC: CheckMate 078 Randomized Phase III Clinical Trial. J Thorac Oncol 2019;14:867-75

38. Fehrenbacher L, Spira A, Ballinger M, et al. Atezolizumab versus docetaxel for patients with previously treated non-small-cell lung cancer (POPLAR): a multicentre, open-label, phase 2 randomised controlled trial. Lancet 2016;387:1837-46.

39. Fehrenbacher L, von Pawel J, Park K, et al. Updated efficacy analysis including secondary population results for OAK: a randomized phase iii study of atezolizumab versus docetaxel in patients with previously treated advanced non-small cell lung cancer. J Thorac Oncol 2018;13:1156-70.

40. Herbst RS, Baas P, Kim DW, et al. Pembrolizumab versus docetaxel for previously treated, PD-L1-positive, advanced non-small-cell lung cancer (KEYNOTE-010): a randomised controlled trial. Lancet 2016;387:1540-50.

41. Barlesi F, Vansteenkiste J, Spigel D, et al. Avelumab versus docetaxel in patients with platinum-treated advanced non-small-cell lung cancer (JAVELIN Lung 200): an open-label, randomised, phase 3 study. Lancet Oncol 2018;19:1468-79.

42. Langer CJ, Gadgeel SM, Borghaei H, et al. Carboplatin and pemetrexed with or without pembrolizumab for advanced, non-squamous non-small-cell lung cancer: a randomised, phase 2 cohort of the open-label KEYNOTE-021 study. Lancet Oncol 2016;17:1497-508.

43. Reck M, Mok TSK, Nishio M, et al. Atezolizumab plus bevacizumab and chemotherapy in non-small-cell lung cancer (IMpower150): key subgroup analyses of patients with EGFR mutations or baseline liver metastases in a randomised, open-label phase 3 trial. Lancet Respir Med 2019;7:387-401.

44. Planchard D, Reinmuth N, Orlov S, et al. ARCTIC: durvalumab with or without tremelimumab as third-line or later treatment of metastatic non-small-cell lung cancer. Ann Oncol 2020;31:609-18.

45. Du L, Herbst RS, Morgensztern D. Immunotherapy in Lung Cancer. Hematol Oncol Clin North Am 2017;31:131-41.

46. Boland JM, Kwon ED, Harrington SM, et al. Tumor B7$\mathrm{H} 1$ and $\mathrm{B} 7-\mathrm{H} 3$ expression in squamous cell carcinoma of the lung. Clin Lung Cancer 2013;14:157-63.

47. Pai-Scherf L, Blumenthal GM, Li H, et al. FDA Approval Summary: Pembrolizumab for Treatment of Metastatic Non-Small Cell Lung Cancer: First-Line Therapy and Beyond. Oncologist 2017;22:1392-9.

48. Weinstock C, Khozin S, Suzman D, et al. U.S. Food and Drug Administration Approval Summary: Atezolizumab for Metastatic Non-Small Cell Lung Cancer. Clin Cancer Res 2017;23:4534-9.

49. Lumley T. Network meta-analysis for indirect treatment comparisons. Stat Med 2002;21:2313-24.

50. Salanti G, Ades AE, Ioannidis JP. Graphical methods and numerical summaries for presenting results from multipletreatment meta-analysis: an overview and tutorial. J Clin Epidemiol 2011;64:163-71.

51. Wang XJ, Lin JZ, Yu SH, et al. First-line checkpoint inhibitors for wild-type advanced non-small-cell cancer: a pair-wise and network meta-analysis. Immunotherapy 2019;11:311-20.

52. Dafni U, Tsourti Z, Vervita K, et al. Immune checkpoint inhibitors, alone or in combination with chemotherapy, as first-line treatment for advanced non-small cell lung cancer. A systematic review and network meta-analysis. Lung Cancer 2019;134:127-40.

53. Wang C, Qiao W, Jiang Y, et al. The landscape of 
immune checkpoint inhibitor plus chemotherapy versus immunotherapy for advanced non-small-cell lung cancer: A systematic review and meta-analysis. J Cell Physiol 2020;235:4913-27.

54. Peng TR, Tsai FP, Wu TW. Indirect comparison between pembrolizumab and nivolumab for the treatment of nonsmall cell lung cancer: A meta-analysis of randomized clinical trials. Int Immunopharmacol 2017;49:85-94.

55. Créquit P, Chaimani A, Yavchitz A, et al. Comparative efficacy and safety of second-line treatments for advanced non-small cell lung cancer with wild-type or unknown status for epidermal growth factor receptor: a systematic review and network meta-analysis. BMC Med 2017;15:193.

56. Tan PS, Aguiar P, Jr., Haaland B, et al. Comparative effectiveness of immune-checkpoint inhibitors for previously treated advanced non-small cell lung cancer A systematic review and network meta-analysis of 3024 participants. Lung Cancer 2018;115:84-8.

57. Kim J, Cho J, Lee MH, et al. Relative Efficacy of Checkpoint Inhibitors for Advanced NSCLC According to Programmed Death-Ligand-1 Expression: A Systematic Review and Network Meta-Analysis. Sci Rep 2018;8:11738.

Cite this article as: Liang J, Li M, Sui Q, Hu Z, Bian Y, Huang Y, Zhan C, Jiang W, Wang Q, Tan L. Compare the efficacy and safety of programmed cell death-1 (PD-1) and programmed cell death ligand-1 (PD-L1) inhibitors for advanced non-small cell lung cancer: a Bayesian analysis. Transl Lung Cancer Res 2020;9(4):1302-1323. doi: 10.21037/tlcr-20-192
58. Almutairi AR, Alkhatib N, Martin J, et al. Comparative efficacy and safety of immunotherapies targeting the PD-1/PD-L1 pathway for previously treated advanced non-small cell lung cancer: A Bayesian network metaanalysis. Crit Rev Oncol Hematol 2019;142:16-25.

59. Antonia S, Goldberg SB, Balmanoukian A, et al. Safety and antitumour activity of durvalumab plus tremelimumab in non-small-cell lung cancer: a multicentre, phase $1 \mathrm{~b}$ study. Lancet Oncol 2016;17:299-308.

60. Hellmann MD, Rizvi NA, Goldman JW, et al. Nivolumab plus ipilimumab as first-line treatment for advanced nonsmall-cell lung cancer (CheckMate 012): results of an open-label, phase 1, multicohort study. Lancet Oncol 2017;18:31-41.

61. Ready N, Hellmann MD, Awad MM, et al. First-Line Nivolumab Plus Ipilimumab in Advanced Non-SmallCell Lung Cancer (CheckMate 568): Outcomes by Programmed Death Ligand 1 and Tumor Mutational Burden as Biomarkers. J Clin Oncol 2019;37:992-1000.

62. Hellmann MD, Ciuleanu TE, Pluzanski A, et al. Nivolumab plus Ipilimumab in Lung Cancer with a High Tumor Mutational Burden. N Engl J Med 2018;378:2093-104. 


\section{Supplementary}
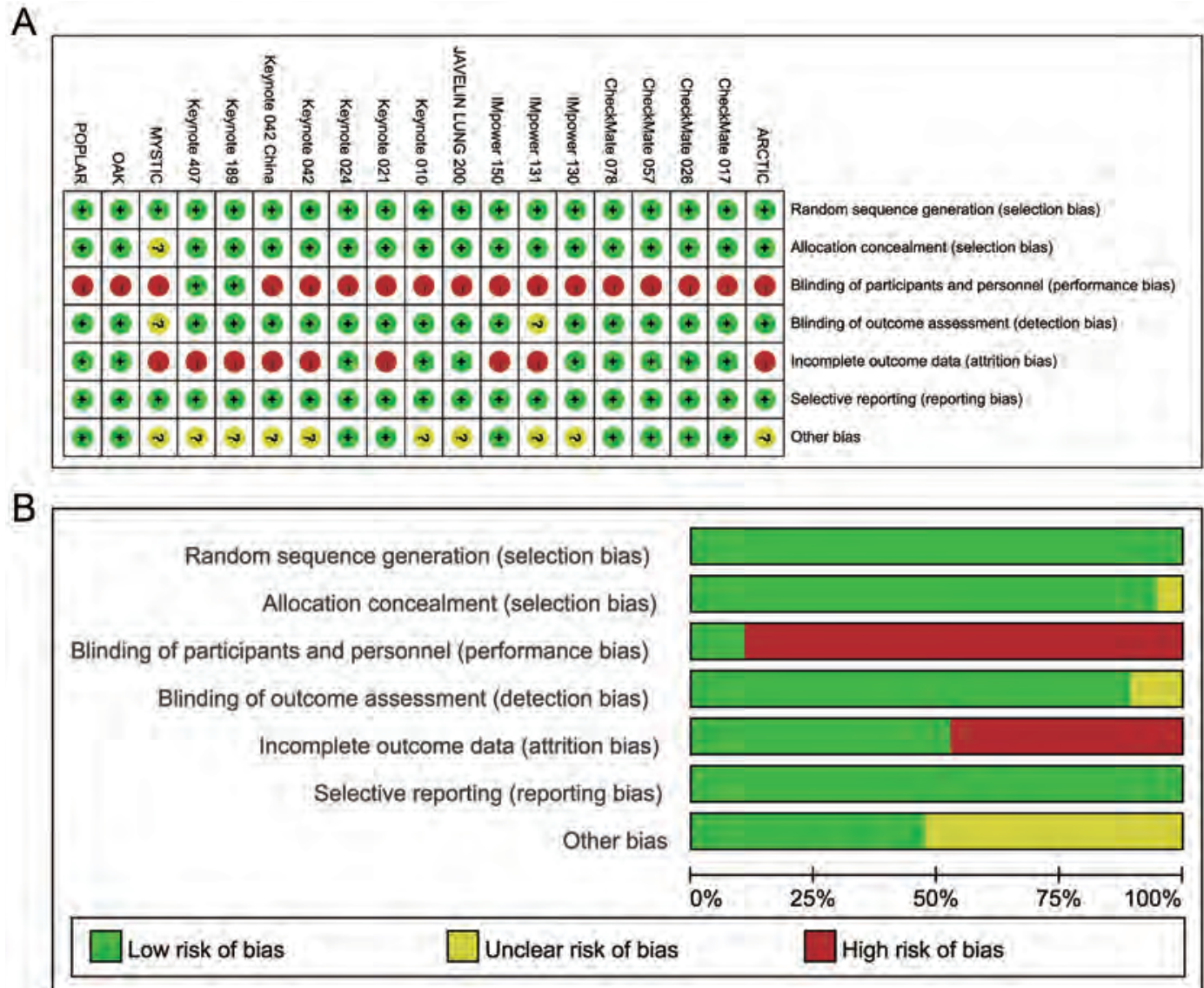

Figure S1 Risk of bias assessment. (A) Risk of bias in individual studies; (B) risk of bias across included studies. 
A

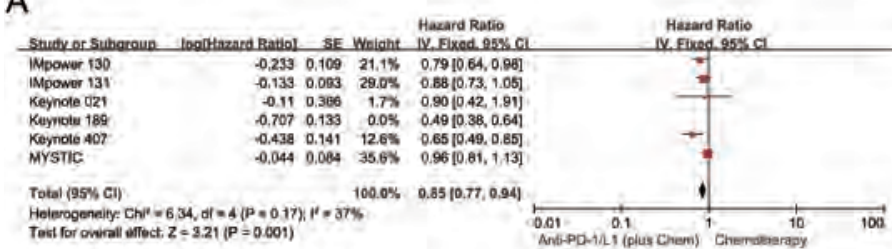

Tost

B

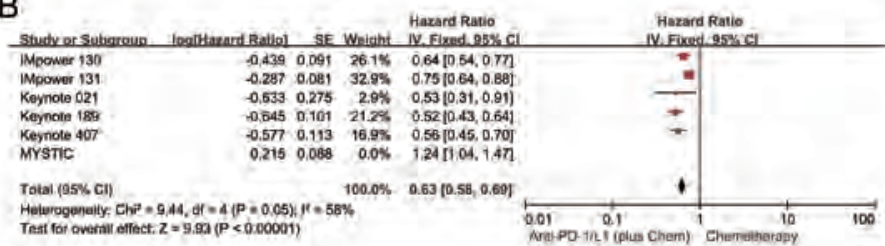

C

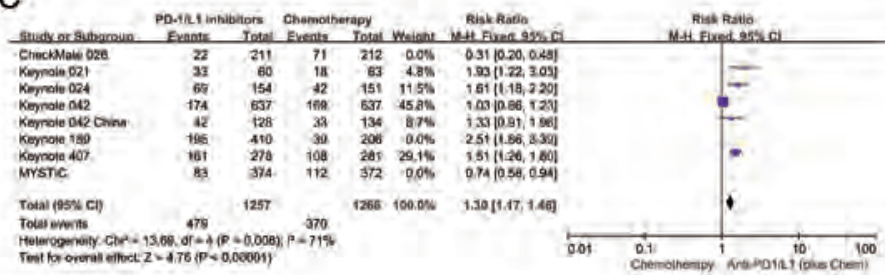

D

D Mazard Ratio

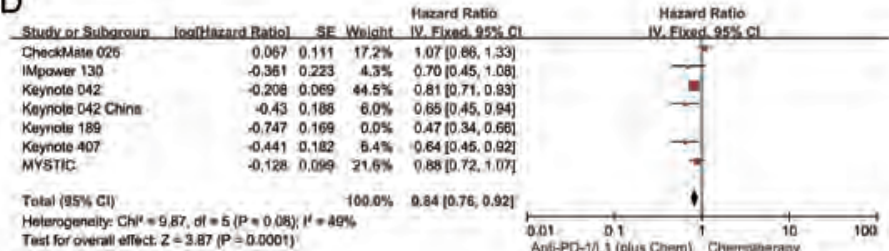

$E$

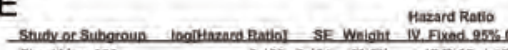

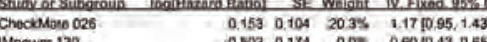
Keynote 0420 $\begin{array}{llllllll} & \text { Keynote } 042 \text { China } & 0.158 & 0.206 & 5.25 & 0.8510 .57 .1 .29\end{array}$ Keynote $189=0.320 .132 \quad 0.05 \quad 0.44[0.34,0.57]$ $\begin{array}{lrrrrr}\text { Keynote } 407 & -0.609 & 0.137 & 0.0 \% & 0.50[0.38,0.65] \\ \text { MYBTIC } & 0.00 & 0.103 & 20.74 & 1.09[0.88,1.34]\end{array}$

Total (95\% C1) 100.

Helerogennity, $C h P=1.69, a t=3(P=0.60) ; t=0 \%$

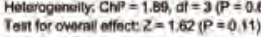

$10.0 \% \quad 1.08(0.98,1.98$

F

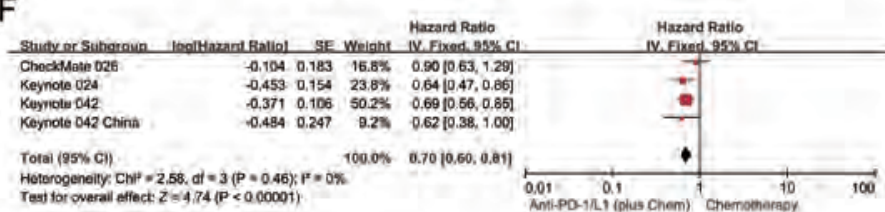

G

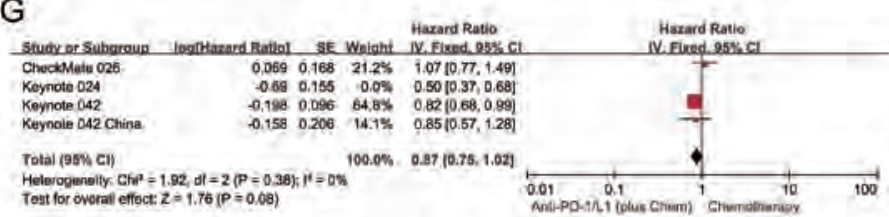

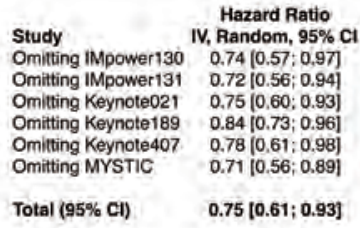

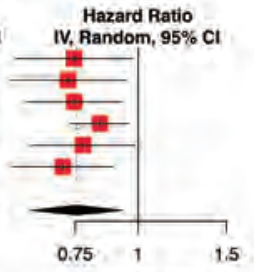

Hazard Ratio

Omitting IV, Random, 95\% CI

Omilting IMpower131 0.67 10.46:0.96!

Omitting Keynote021 0.71 . $0.52,0.961$

Omitting Keynote189

Omitting Keynote 407

Omitting MYSTIC

$0.7310 .53 ; 0.99$

$0.73[0.53 ; 0.99]$

$0.71[0.52 ; 0.98]$
$0.61[0.53 ; 0.71]$

Total $(95 \% \mathrm{CI})$

$0.68[0.52 ; 0.91]$

$\begin{array}{ll}\text { Hazard Ratio } \\ \text { Study } & \text { IV, Random, 95\% }\end{array}$

Omitting CheckMate026 IV, Random, 95\%

Omituing Keynote021 1.11 1.76;1.63)

Omiting Keynoleo24

Omitting Keynote042 China

Omitting Keynote189

Omitting Keynote 407
Omitting MYSTIC

Total $(95 \%$ Cl) $1.11[0.76 ; 1.63]$ $1.21[0.78 ; 1.87]$ $1.21[0.78 ; 1.87]$ $1.17[0.79: 1.73]$ $1.07(0.75 ; 1.50]$ $1.14[0.75 ; 1.75]$ $1.27[0.88 ; 1.85]$

$1.19[0.83 ; 1.69]$

Hazard Ratio

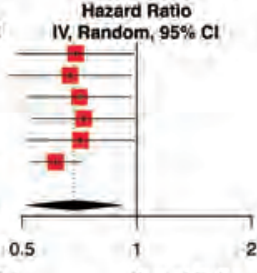

Hazard Fatio

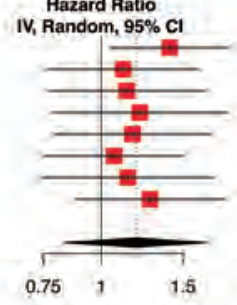

Study

Omitting CheckMate026

Omitting IMpower 130

Omitting Keynote 042

Omitting Keynote $042 \mathrm{Ch}$

Omitting Keynote 189

Omitting Keynote407

Omitting MYSTIC

Total $(95 \% \mathrm{CI})$

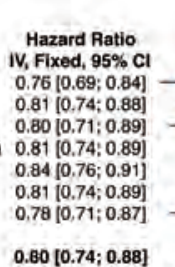

Hazard Ratio

IV. Fixed, $95 \% \mathrm{C}$
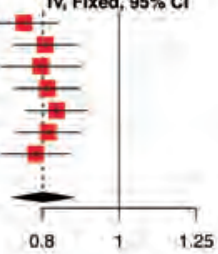

Study

Omitting CheckMate026

Omitting IMpower130

Omitting Keynote042

Omitting Keynote 042 Chin

Omitting Keynote 189

Omitting Keynote407

Omitting MYSTIC

Total $(95 \%$ CI)
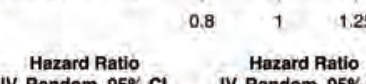

, Random, 95\% $0.72[0.51 ; 1.01]$ $0.73[0.50 ; 1.05]$

$0.76[0.55 ; 1.06]$

$0.86(0.66 ; 1.11$

$0.83[0.62 ; 1.12$

$0.73[0.51: 1.03]$

$0.77[0.57 ; 1.04]$

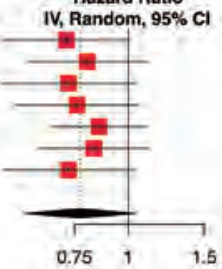

Study

Omitting CheckMate026

Omitting Keynole024

Omitting Keynote 042

Total $(95 \% \mathrm{cl})$

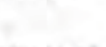

0.7511 .5

Hazard Ratio IV, Fixed, 95\% CI $0.67[0.57 ; 0.78]$
$0.72[0.61 ; 0.86]$

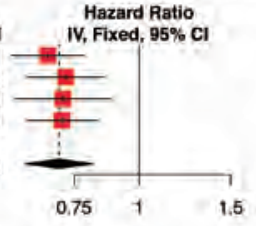

Study

Omitting CheckMate026

Omilting Keynole024

Omitting Keynole042

Omitting Keynote042 China

Total $(95 \% \mathrm{CI})$
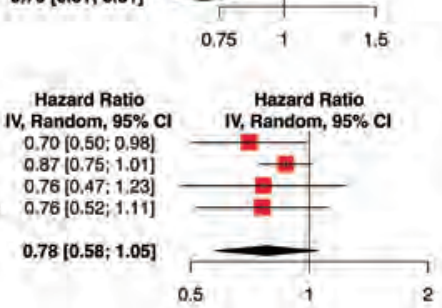

Figure S2 Forest plots and sensitivity analyses of efficacy analyses with PD-1/L1 inhibitors in the first-line settings. (A) Overall survival (OS) in the intention-to-treat (ITT) patient population; (B) progression-free survival (PFS) in the ITT patient population; (C) objective response rate (ORR) in the patient population who had received more than one dose of treatment; (D) OS in the patient population with tumor proportion score (TPS) of PD-L1 $\geq 1 \%$; (E) PFS in the patient population with PD-L1 TPS $\geq 1 \%$; (F) OS in the patient population with PD-L1 TPS $\geq 50 \%$; (G) PFS in the patient population with PD-L1 TPS $\geq 50 \%$. 

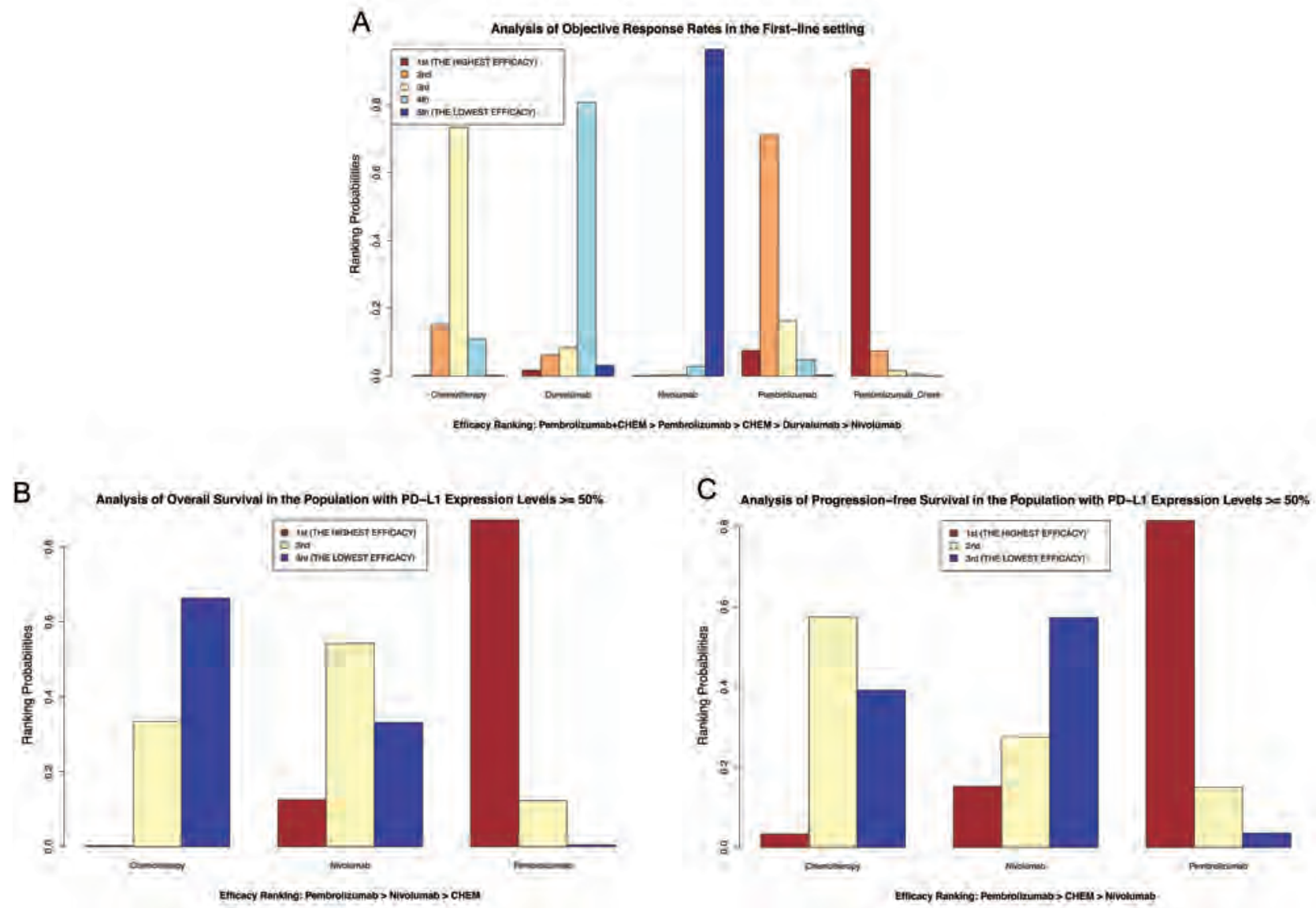

Figure S3 Rankograms of efficacy analyses with PD-1/L1 inhibitors. (A) Objective response rate (ORR) in the patient population who had received more than one dose of treatment in the first-line settings; (B) overall survival (OS) in the patient population with PD-L1 TPS $\geq 50 \%$ in the first-line settings; (C) progression-free survival (PFS) in the patient population with PD-L1 TPS $\geq 50 \%$ in the first-line settings. 
A
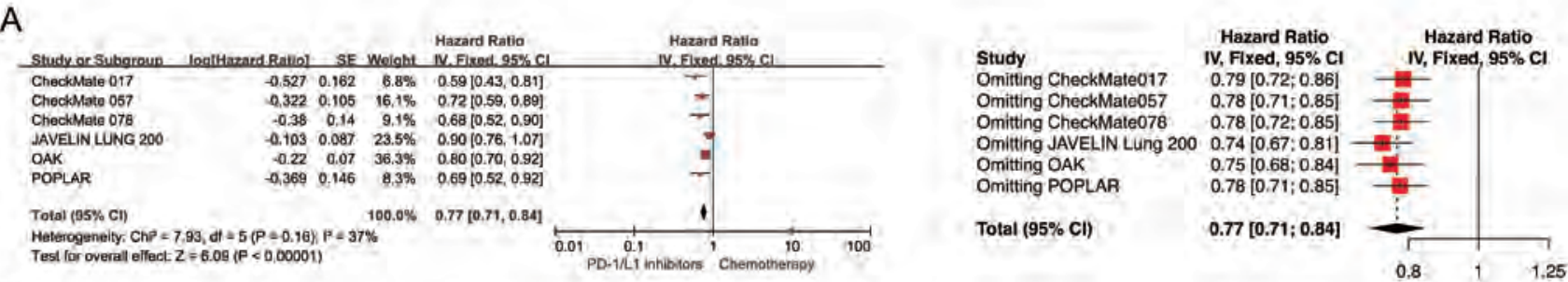

B
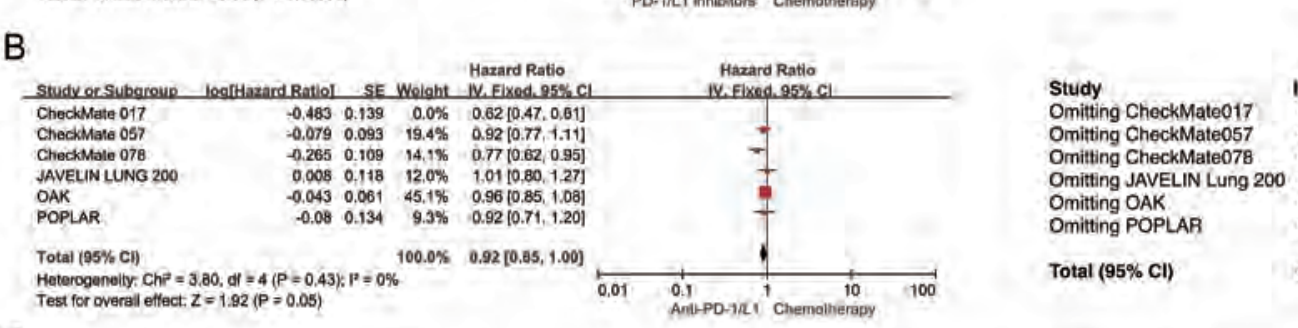

C

C
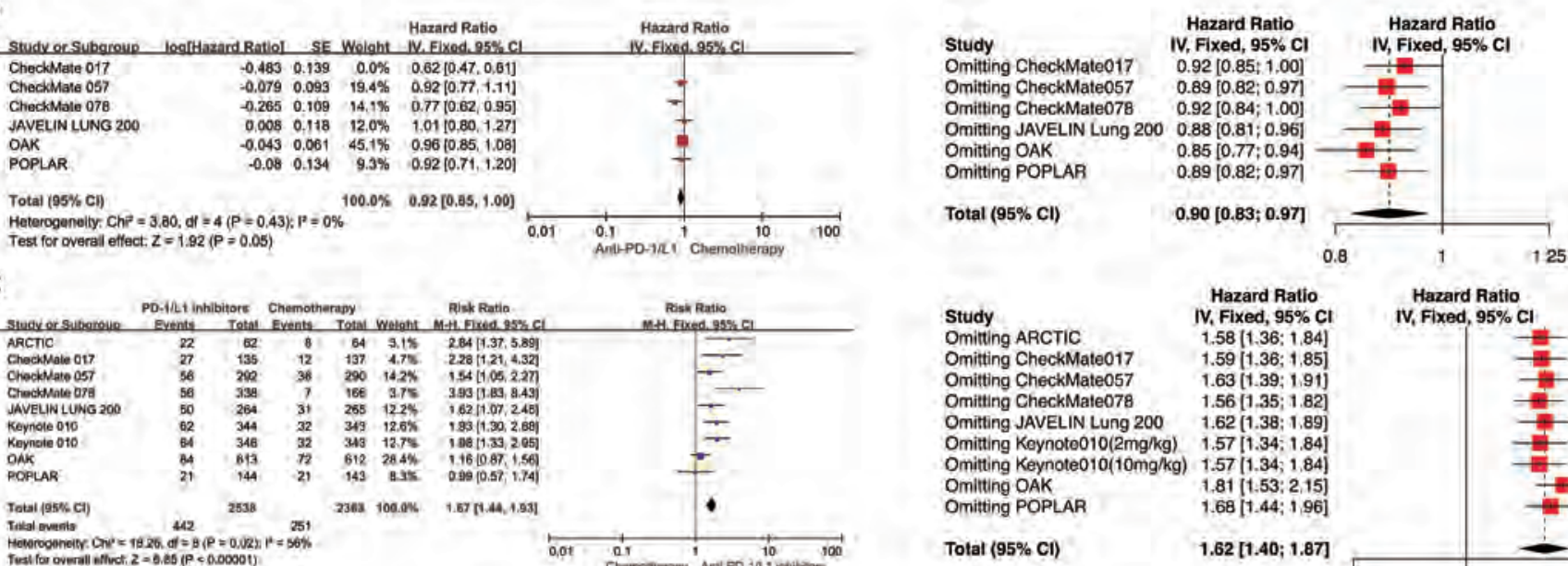

D Mazard Ratio

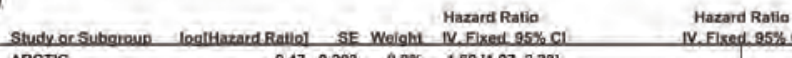
CheckMate 017 CheckMate 057 CheckMati 078 JAVELIN LUNG Keynote 010 Keynole 010

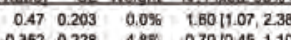
$\begin{array}{lllll}-0.352 & 0.228 & 4.8 \% & 0.7010 .45,1.10\end{array}$ $\begin{array}{llll}-0.533 & 0.171 & 8.5 \% & 0.59(0.42,0.82\end{array}$ $\begin{array}{lllll}-0.469 & 0.168 & 8.8 \% & 0.63[0.45,0.87)\end{array}$ $\begin{array}{llll}-0.105 & 0.107 & 21.7 \% & 0.90[0.73,1.11]\end{array}$

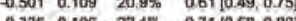
$\left.\begin{array}{llll}-0.444 & 0.137 & 13.2 \% & 0.64[0.49,0.84]\end{array}\right]$

Total $(95 \%$ CI)

$100.0 \% \quad 0.69[0.63,0.77$ Hetor che $=9.22$, df $=6 .(P=0.16) ; P=35 \%$ Test for overall leffect: $Z=7,30,(P<0.00001)$

E

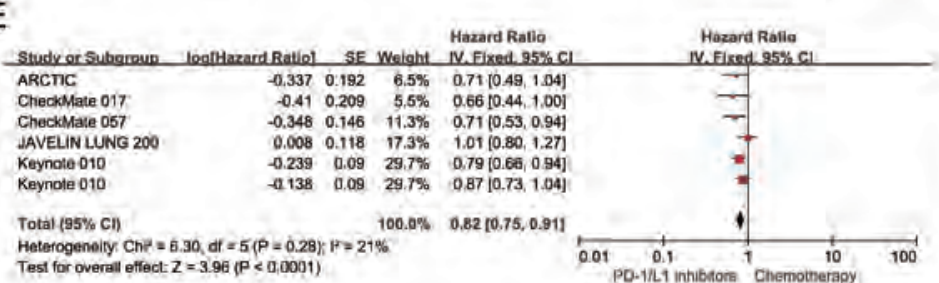

Study

Omitting ARCTIC

Omitting CheckMale017

Omitting CheckMate057

Omitting CheckMate078

Omitting JAVELIN Lung 200

Omititing Keynote010(2mg/kg)

Omitting Keyno

Omitting OAK

Total $(95 \% \mathrm{Cl})$

\section{Study}

Omitting AACTIC

Omitting CheckMale017

Omitring CheckMale057

Omitting JAVELIN Lung 200

Omitting Keynote010(2mg/kg)

Omitting Keynote010(10mg/kg)

Total $(95 \% \mathrm{Cl})$
Hazard Ratio IV. Fixed, $95 \%$ 1.58 [1.36; 1.84$]$ $1.59[1.36 ; 1,85]$ $1.63[1.39 ; 1.91]$ $1.56[1.35 ; 1.82]$ 1.57 (1.34:1.84] 1.81 11.53:215 $1.68[1.44 ; 1,96]$

$1.62[1.40 ; 1.87]$

\section{Hazard Ratio-} IV, Fixed, 95\% C $0.70[0.63: 0.77]$ $0.73(0.66 ; 0.80$ $0.74[0.67 ; 0.82]$ $0.74(0.67 ; 0.82)$ $0.69[0.62 ; 0.77]$ $0.76[0.68 ; 0.85)$ $0.740 .66: 0.82$ $0.73[0.66 ; 0.80]$

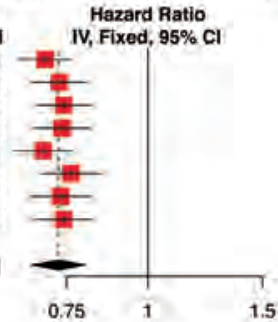

Hazard Ratio V, Fixed, 95\% C $0.83[0.75 ; 0.92]$ $0.83[0.76 ; 0.92]$ $0.84[0.76 ; 0.93)$ $0.79[0.71 ; 0.88]$ $0.84[0.75 ; 0.94$ $0.81[0.72 ; 0.90]$

$0.82[0.75 ; 0.91]$

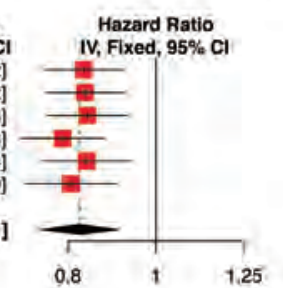

Figure S4 Forest plots and sensitivity analyses on efficacy with PD-1/L1 inhibitors in the first-line settings. (A) Overall survival (OS) in the intention-to-treat (ITT) patient population; (B) progression-free survival (PFS) in the ITT patient population; (C) objective response rate (ORR) in the patient population who had received more than one dose of treatment; (D) OS in the patient population with tumor proportion score (TPS) of PD-L1 $\geq 1 \%$; (E) PFS in the patient population with PD-L1 TPS $\geq 1 \%$. 


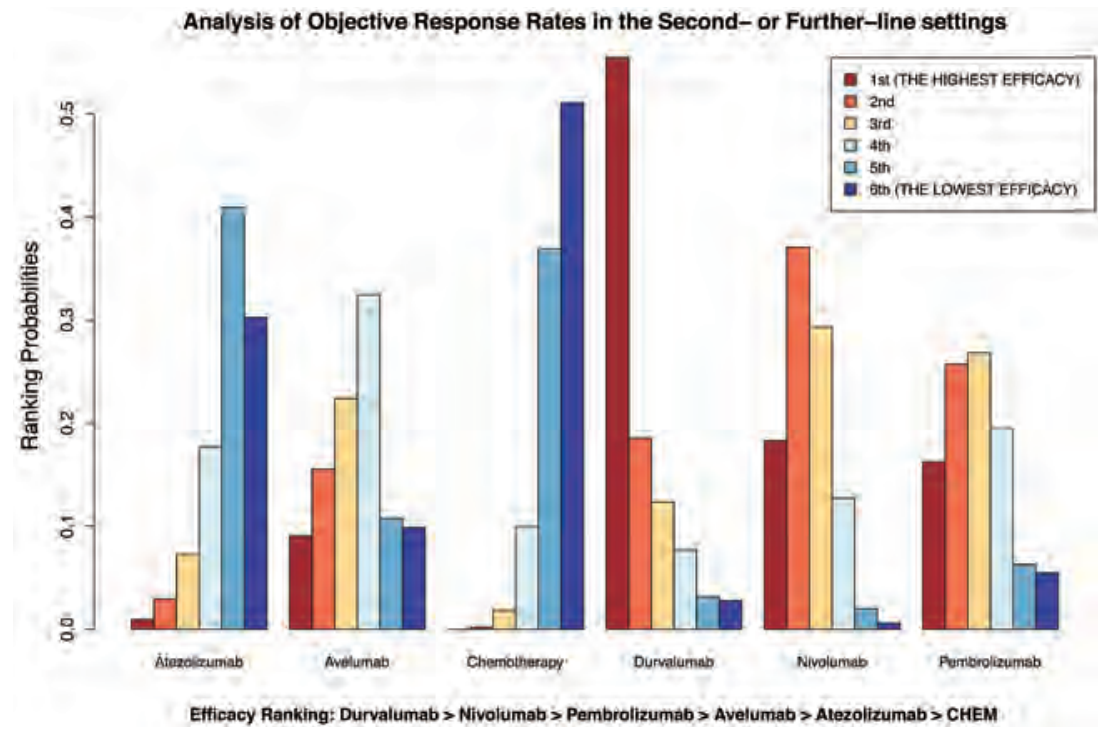

Figure S5 Objective response rate (ORR) in the patient population who had received more than one dose of treatment in the second- or further-line settings. 
A

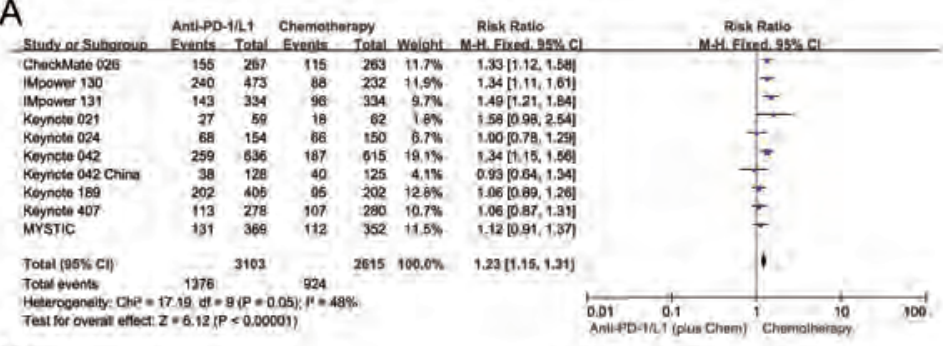

B

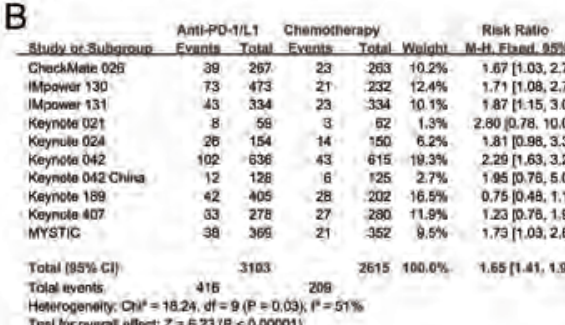

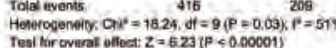

c

C

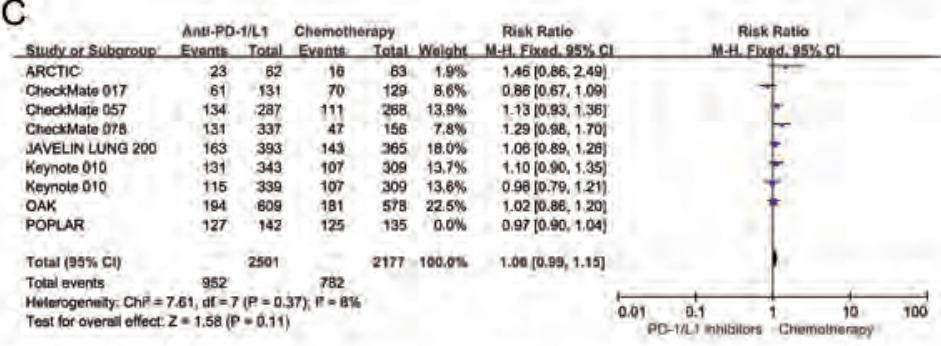

D
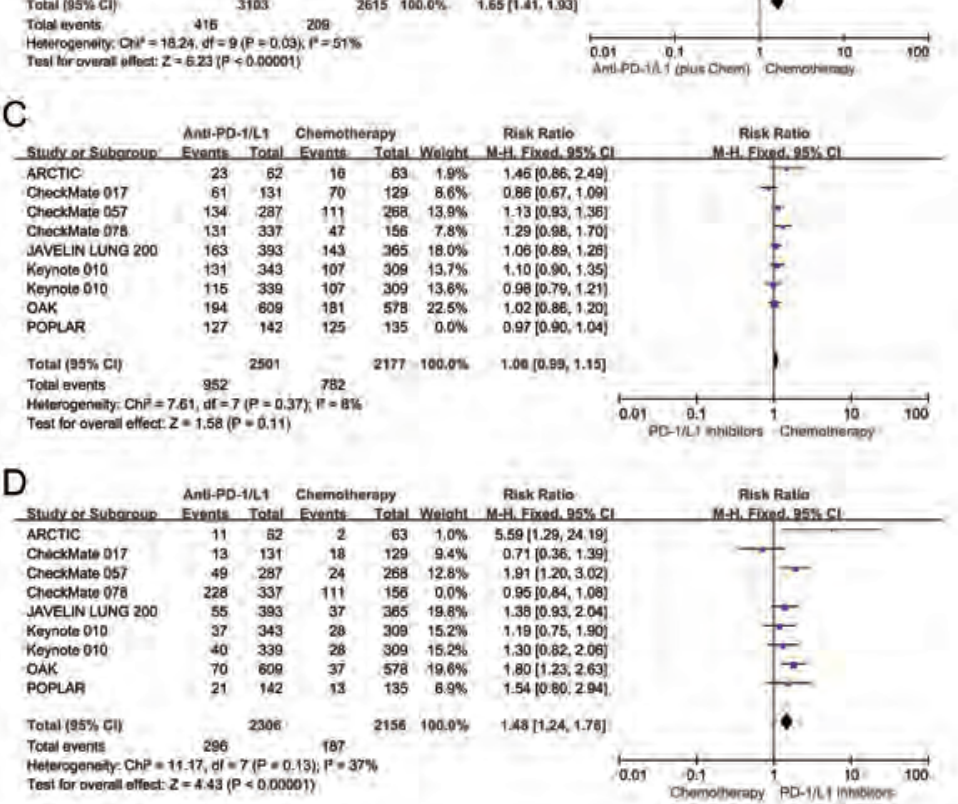

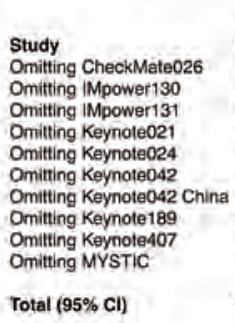

Hazard Ratio

IV, Fixed, 95\% C|

$20[1.12 ; 1.29]$

$1.21[1.12 ; 1.29]$

$1.20[1.12 ; 1.28]$

$1.21[1.14 ; 1.30]$ $.24[1.16 ; 1.32]$ .20 [1.11: 1.28$]$ $23[1.15 ; 1.32]$ $25[1.16 ; 1.34]$ $24[1.16 ; 1.33]$ $1,23[1,15 ; 1.32]$

$1.22[1.14 ; 1.30]$

Study

Omitting CheckMate026

Omitting IMpower130

Omitting iMpower131

Omitting Keynote021

Omitting Keynote024

Omitting Keynote042 Ch

Omitting Keynote189

Omitting Keynotes0

Total $(95 \% \mathrm{CI})$

Hazard Ratio

IV, Fixed, 95\% Cl

$1.61[1.36 ; 1.91]$

$1.61[1.36 ; 1.91]$

1.59 [1.35; 1.88$]$

$1.61[1.37 ; 1.89]$

$1.61[1.36 ; 1.90]$

1.47 [1.23; 1.76]

$1.61[1.37 ; 1.89]$

$1.81[1.53 ; 2.15]$

$1.68[1.42 ; 1.98]$

$1.61[1.36 ; 1.90]$

$1.62[1.38 ; 1.90]$

$62[1.38 ; 1.90]$
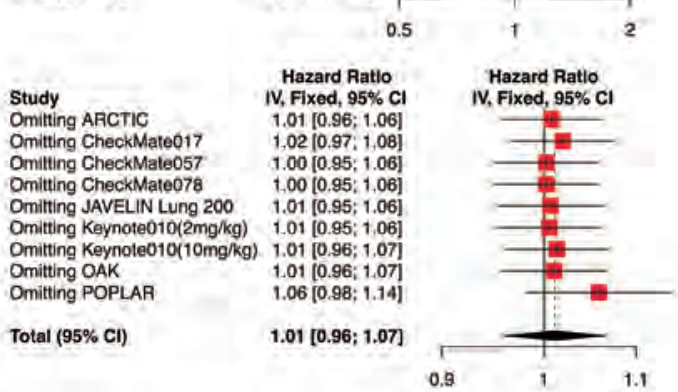

Study

Hazard Ratio

Omitting CheckMate017

Omitting CheckMate057

Omitting CheckMate078
Omitting JAVELIN Lung 200

Omitting Keynote010(2mg/kg)

Omitting Keynote010(10mg/kg)

Omitting OAK

Omitting POPLAR

$$
\text { IV }
$$

V, Random, $95 \%$ CI

$1.24[1.00 ; 1.54]$

1.34 [1.06; 1.70$]$

$1.30[1.01: 1.67]$

1.38 [1.14: 1.68]

$1.27[0.99 ; 1.64]$

1.30 [1.01; 167$]$

$1.28[1.00 ; 1.65]$
$1.22[0.97 ; 1.53]$

$1.24[0.97 ; 1.58]$

Total $(95 \% \mathrm{Cl})$

1.28 [1.02; 1.61]

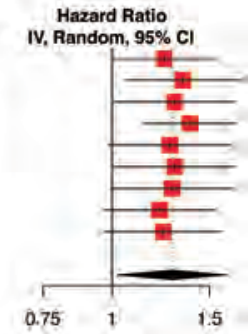

Figure S6 Forest plots and sensitivity analyses on safety with PD-1/L1 inhibitors. (A) Incidence of severe adverse events (SAEs) in the firstline settings; (B) incidence of respiratory and thoracic disorders in the first-line settings; (C) incidence of SAEs in the second- or further-line settings; (D) incidence of respiratory and thoracic disorders in the second- or further-line settings.

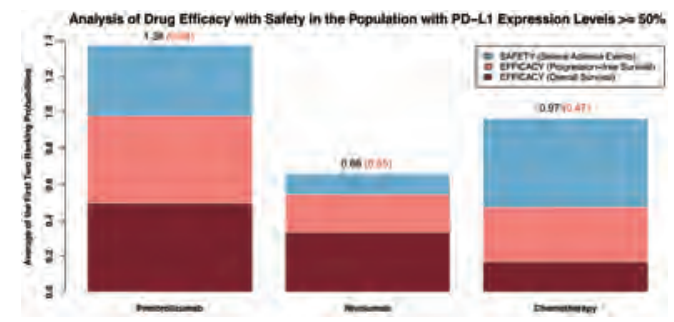

Figure $\mathbf{S} 7$ The stacked bar chart of the average of first two leading ranking probabilities with $\mathrm{PD}-1 / \mathrm{L} 1$ inhibitors. Accumulated probabilities in terms of overall survival (OS), progression-free survival (PFS), and the incidence of severe adverse events (SAEs) in the patient population with tumor proportion score (TPS) of PDL1 $\geq 50 \%$ in the first-line settings. 